\title{
The Effects of Antipsychotics on the Synaptic Plasticity Gene Homer1a Depend on a Combination of Their Receptor Profile, Dose, Duration of Treatment, and Brain Regions Targeted
}

\author{
Felice Iasevoli ${ }^{1}$, Elisabetta Filomena Buonaguro ${ }^{1}$, Camilla Avagliano ${ }^{1}$, Annarita Barone ${ }^{1}$ (i), \\ Anna Eramo ${ }^{2}$, Licia Vellucci ${ }^{1}$ and Andrea de Bartolomeis ${ }^{1, *}$ \\ 1 Laboratory of Molecular and Translational Psychiatry and Unit of Treatment Resistant Psychosis, \\ Section of Psychiatry, Department of Neuroscience, Reproductive Science, and Odontostomatology, \\ University of Naples Federico II, 80131 Napoli, Italy; felice.iasevoli@unina.it (F.I.); \\ lisabuonaguro@hotmail.com (E.F.B.); camilla.avagliano@gmail.com (C.A.); \\ annaritabarone1@gmail.com (A.B.); liciavellucci2@gmail.com (L.V.) \\ 2 Lundbeck LLC, Deerfield, IL 60015, USA; AERA@Lundbeck.com \\ * Correspondence: adebarto@unina.it
}

Received: 6 June 2020; Accepted: 31 July 2020; Published: 3 August 2020

\begin{abstract}
Background: Antipsychotic agents modulate key molecules of the postsynaptic density (PSD), including the Homer1a gene, implicated in dendritic spine architecture. How the antipsychotic receptor profile, dose, and duration of administration may influence synaptic plasticity and the Homer1a pattern of expression is yet to be determined. Methods: In situ hybridization for Homer1a was performed on rat tissue sections from cortical and striatal regions of interest (ROI) after acute or chronic administration of three antipsychotics with divergent receptor profile: Haloperidol, asenapine, and olanzapine. Univariate and multivariate analyses of the effects of topography, treatment, dose, and duration of antipsychotic administration were performed. Results: All acute treatment regimens were found to induce a consistently higher expression of Homer1a compared to chronic ones. Haloperidol increased Homer1a expression compared to olanzapine in striatum at the acute time-point. A dose effect was also observed for acute administration of haloperidol. Conclusions: Biological effects of antipsychotics on Homer1a varied strongly depending on the combination of their receptor profile, dose, duration of administration, and throughout the different brain regions. These molecular data may have translational valence and may reflect behavioral sensitization/tolerance phenomena observed with prolonged antipsychotics.
\end{abstract}

Keywords: psychosis; synaptic plasticity; gene expression; dopamine; glutamate; haloperidol; asenapine; olanzapine; treatment resistant schizophrenia

\section{Introduction}

Antipsychotics are considered the gold-standard treatment for psychosis [1]. Despite the well-known involvement of dopamine D2 receptor (D2R) occupancy in the antipsychotic mechanism of action [2,3], and the demonstrated effects on synaptic plasticity and metaplasticity [4], there is still a dearth of information on some basic aspects regarding the antipsychotic impact on the synapse. How antipsychotic dose, receptor profile, duration of administration, and brain region targeted by each agent may influence synaptic plasticity is yet to be determined. A better understanding of these variables in antipsychotic effects may also influence our knowledge of the mechanism responsible for the loss or reduction of efficacy over time of antipsychotics treatment. 
Notably, antipsychotic agents have been found to modulate the expression of glutamatergic genes of the postsynaptic density (PSD), an electron-dense thickening at the surface of the glutamatergic postsynaptic membrane [5-7], actively involved in behavioral disorders' molecular pathophysiology [8,9]. Among the most replicated findings, antipsychotics have been found to modulate the immediate-early gene Homer1a [10-12], whose expression has been demonstrated to sculpt the dendritic spine and overall synaptic plasticity $[5,13]$.

Moreover, Homer has been per se considered a candidate gene in schizophrenia and other psychiatric diseases [14-18] and has been demonstrated to be involved in animal models of behavioral disorders, including schizophrenia [19-25]. The Homer1a gene codes for the truncated isoform of the long Homer1 protein. Once expressed, Homer1a competes with the long isoforms and disassembles protein clusters formed by the long Homer isoforms and multiple PSD targets, including PSD-95, Shank, and mGluR5 [26]. As a result of Homer1a induction, the synaptic microdomain in which Homer proteins operate undergoes multiple functional and architectural rearrangements, including changes in intracellular $\mathrm{Ca}^{++}$dynamics [27], shifts in surface channel functioning [28], and modifications in synaptic ultrastructure [29]. It modulates the interaction between metabotropic glutamatergic receptors (mGluRs) and intracellular effectors [30], thereby modulating the strength and direction of intracellular signaling [31]. Changes in Homer1a levels within the PSD may, in turn, affect the reciprocal interaction between NMDA and mGluRs, and regulate surface channel activity [28,32]. Given the complexity of Homer1a biological action, which is intrinsic to synaptic plasticity, the antipsychotic liability of modulating the expression of this gene appears relevant to these agents' molecular actions.

In previous reports, indirect and only partially informative evaluations of outcomes of the divergent duration of treatments (i.e., acute vs. chronic effects) on Homer1a expression were provided [33-35]. A direct and systematic evaluation of the combined effects of the dose and receptor profile has not been carried out yet. Based on all these considerations, we wanted to test the hypothesis that the putative effects of antipsychotics on the Homer1a gene expression dose (or antipsychotic receptor profile) may be differentially mediated by the dose, receptor profile, duration of treatment, and regions of interest at which the gene expression was evaluated.

With the scope of tackling these issues, univariate analyses were run to assess independent time, dose, or receptor profile effects within each region of interest (ROI). Furthermore, as a secondary goal, multivariate analyses were run to study the patterns of Homer1a mRNA expression throughout multiple cortical and striatal regions of interest after acute (i.e., single administration) or chronic (21-day administration period) treatment regimens.

The dose effect was investigated by incremental doses of the high multi-receptor second-generation antipsychotic asenapine, characterized by the breadth of activities at multiple serotonergic receptors $[36,37]$. To investigate the dose effect by a first-generation narrower receptor profile agent, in a separate set of analyses, we compared gene expression by three different doses of the relatively selective D2R blocker haloperidol. In the third set of analysis, we investigated the receptor profile effect on gene expression by comparing asenapine, haloperidol, and olanzapine at behaviorally and neurochemically comparable doses. Olanzapine was chosen as a reference second-generation antipsychotic, with a receptor profile and biological effects that may be considered sharply divergent from asenapine [38].

Interestingly, these three compounds exhibit significantly different D2/D1 ratios (25:1 for haloperidol, 3:1 for olanzapine, and approximately 1:1 for asenapine) [38-40] and this feature may be relevant in order to understand the contribution of the dopaminergic antagonism in triggering the transcription of key molecules of synaptic architecture, such as Homer1a. In fact, as well as D2R-blockers, even selective D1R antagonists have been found to influence striatal Homer1a expression considerably [41]. Moreover, asenapine acts as a more potent antagonist in particular at 5-HT1A and 5-HT2 receptors ( $\mathrm{pKB}=7.4$ for 5-HT1A; $\mathrm{pKB}=9$ for 5 -HT2), in comparison to olanzapine ( $\mathrm{pKB}<5$ for both 5-HT1A and 5-HT2) [38]. Testing an antipsychotic agent with such remarkable serotonergic action was even more appealing for their potential to induce cortical Homer1a expression [35]. Therefore, although olanzapine and asenapine both belong to the class of atypical agents and show a complex, 
partially overlapping, multireceptor binding profile, they may widely differ in terms of glutamatergic activity and capability to affect synaptic plasticity.

\section{Results}

A three-way mixed ANOVA was run to understand the effects of ROI, treatment, and time on Homer1a mRNA expression. Data are the mean fold changes of the relative disintegration per minutes (DPM) compared to the vehicle \pm standard error mean. Shapiro-Wilk's test confirmed that the relative DPM values were normally distributed (Tables S1-S6). Levene's test for equality of variances showed homogeneity of variances for the relative DPM throughout all ROIs. Outputs using raw (i.e., non-relativized) data are reported in Tables S7-S10.

\subsection{Paradigm 1}

In this paradigm, the three categorical independent variables were: Treatment with incremental asenapine doses (thereafter asenapine Doses); time; and ROIs. The asenapine doses variable comprised three categories: $0.05 \mathrm{mg} / \mathrm{kg}$ treatment group; $0.1 \mathrm{mg} / \mathrm{kg}$ treatment group; and $0.3 \mathrm{mg} / \mathrm{kg}$ treatment group. The time variable comprised two categories: Acute administration and chronic administration. The ROIs variable comprised five categories in the cortex (anterior cingulate cortex, ACC; medial agranular cortex, MAC; motor cortex, MC; somatosensory cortex, SS; insular cortex, IC) and six categories in striatum (dorso-medial caudate putamen, DM; dorso-lateral caudate putamen, DL; ventro-medial caudate putamen, VM; ventro-lateral caudate putamen, VL; nucleus accumbens core, $\mathrm{CAb}$; nucleus accumbens shell, Sab; Figure 1).

A)

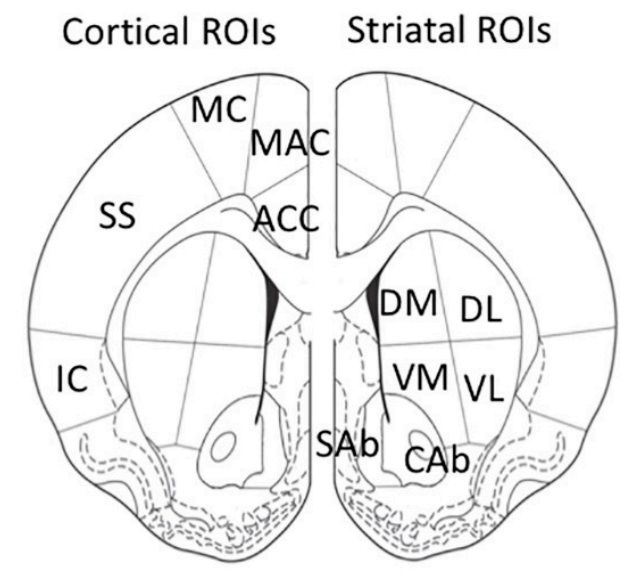

B)

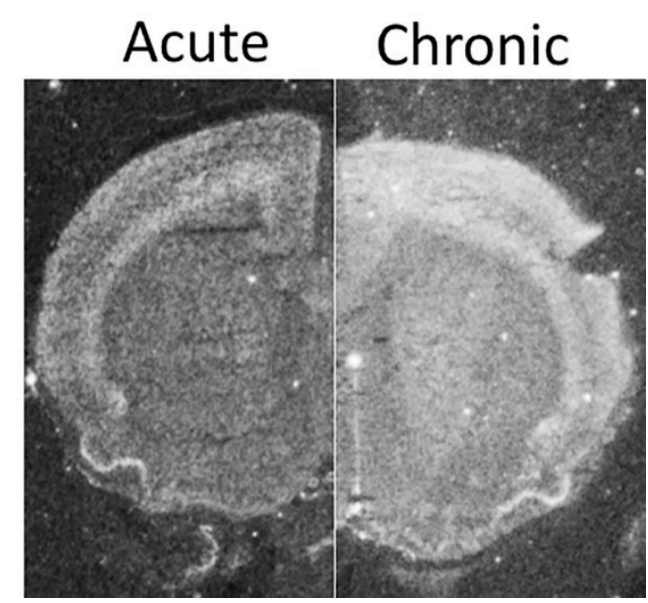

Figure 1. (A) Panel A: diagrams of regions of interest (ROIs) quantitated on autoradiographic film images in the rat forebrain in which gene expression was measured. ACC = anterior cingulated cortex; $\mathrm{MAC}=$ medial agranular cortex; $\mathrm{MC}=$ motor cortex; $\mathrm{SS}=$ somatosensory cortex; $\mathrm{IC}=$ insular cortex; $\mathrm{DM}=$ dorso-medial caudate putamen; $\mathrm{DL}=$ dorso-lateral caudate putamen; $\mathrm{VM}=$ ventro-medial caudate putamen; $\mathrm{VL}=$ ventro-lateral caudate putamen; $\mathrm{Cab}=$ core of the nucleus accumbens; $\mathrm{Sab}=$ shell of the nucleus accumbens. Modified from Paxinos and Watson (1997); (B) Panel B: representative juxtaposed autoradiogram sections from short- (left side) and long-term (right side) treatment by vehicle.

\subsubsection{Striatum}

No significant effect of asenapine doses was found in any striatal ROI at either acute or chronic time point (Table 1, Figure 2). 
a)

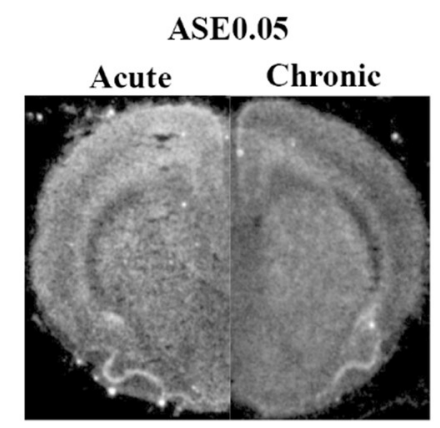

b)
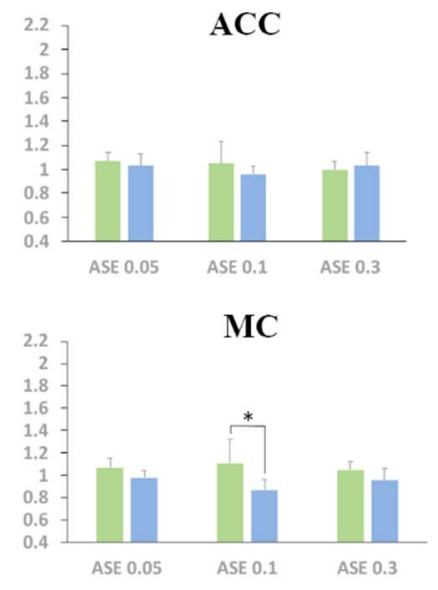

DM

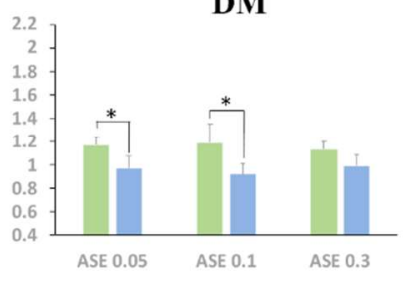

VL

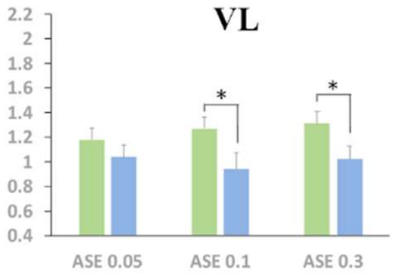

ASE0.1
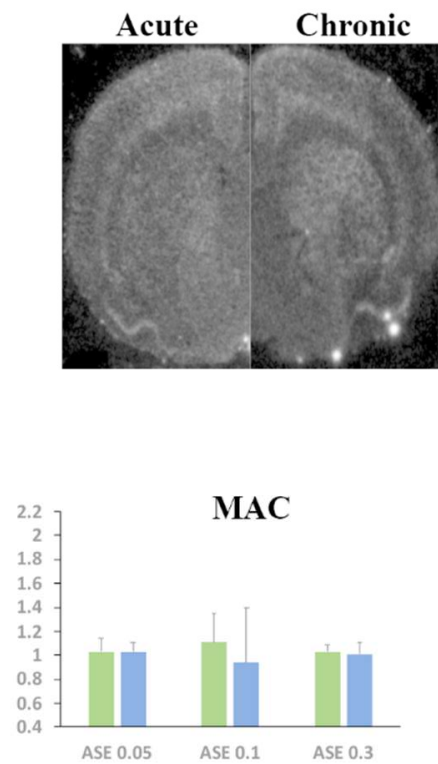

SS

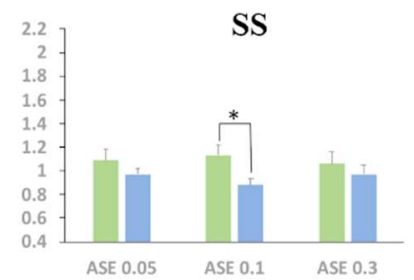

DL

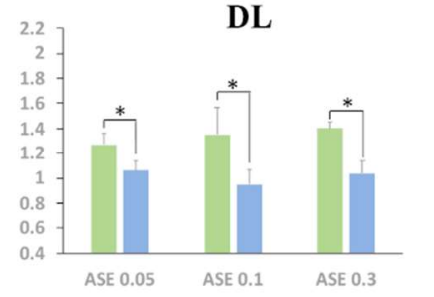

CAb

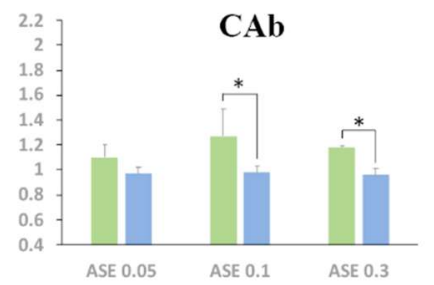

ASE0.3

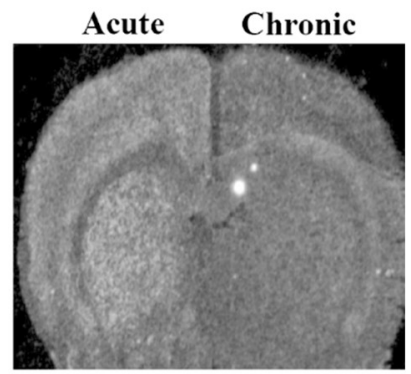

Acute

- Chronic

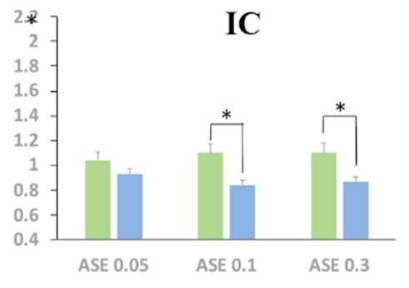

VM

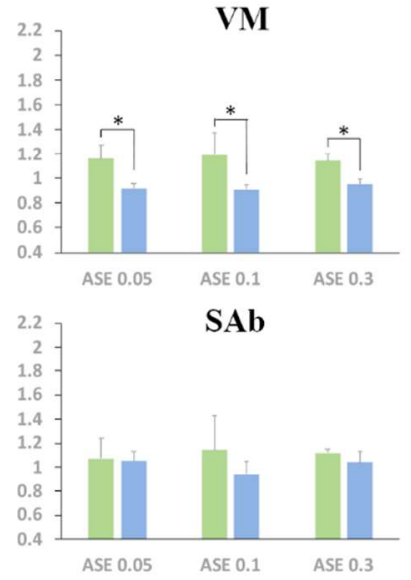

Figure 2. (a) Upper section: representative juxtaposed autoradiogram sections from acute (left side) and chronic (right side) treatment by asenapine at $0.05 \mathrm{mg} / \mathrm{kg}$ (ASE 0.05), $0.1 \mathrm{mg} / \mathrm{kg}$ (ASE 0.1), and $0.3 \mathrm{mg} / \mathrm{kg}$ (ASE 0.3), respectively; (b) Lower section: levels of Homer1a mRNA expression by different doses of asenapine at either acute (green bars) or chronic (blue bars) time points in the cortical and striatal regions of interest (ROIs). Gene expression is given as fold changes of vehicle-induced relative DPMs \pm standard error mean (S.E.M.). ASE0.1 = asenapine at $0.1 \mathrm{mg} / \mathrm{kg}$; ASE0.3 = asenapine at $0.3 \mathrm{mg} / \mathrm{kg}$; ACC = anterior cingulated cortex; $\mathrm{MAC}=$ medial agranular cortex; $\mathrm{MC}=$ motor cortex; $\mathrm{SS}=$ somatosensory cortex; IC = insular cortex; DM = dorso-medial caudate putamen; DL = dorso-lateral caudate putamen; $\mathrm{VM}=$ ventro-medial caudate putamen; $\mathrm{VL}=$ ventro-lateral caudate putamen; $\mathrm{Cab}=$ core of the nucleus accumbens; $\mathrm{Sab}=$ shell of the nucleus accumbens. *: significantly different groups at Student's $\mathrm{t}$ test (time effect). Significantly different groups are connected by brackets.

A statistically significant effect of time was found for all doses in distinct striatal ROIs (Table 1, Figure 2). More specifically, mean Homer1a expression was higher after acute compared to chronic 
administration of: ASE0.05 in DM, DL, and VM; ASE0.1 in all striatal ROIs with the exception of SAb; and ASE0.3 in DL, VM, VL, and CAb (Table 1, Figure 2).

Table 1. Dose and time-independent effects on asenapine-mediated Homer1a expression as assessed by univariate analysis in each region of interest. On the right side of the table, the "dose effect" was analyzed separately for acute and chronic administration regimens, in order to determine if asenapine-mediated Homer1a expression was different for groups exposed to different asenapine doses (i.e., ASE0.05, ASE0.1, and ASE0.3). On the left side of the table, the "time effect" was analyzed separately for each asenapine dose, in order to determine if asenapine-mediated Homer1a expression was different for groups with different durations of treatment (i.e., acute and chronic). Significant effects are given in bold. ASE0.05 = asenapine at $0.05 \mathrm{mg} / \mathrm{kg} ;$ ASE0.1 = asenapine at $0.1 \mathrm{mg} / \mathrm{kg}$; ASE0.3 = asenapine at $0.3 \mathrm{mg} / \mathrm{kg} . \mathrm{ns}=$ not significant.

\begin{tabular}{|c|c|c|c|c|c|c|c|c|c|c|c|}
\hline & & \multicolumn{4}{|c|}{ Dose Effect } & & & \multicolumn{4}{|c|}{ Time Effect } \\
\hline & & \multicolumn{2}{|c|}{$\begin{array}{c}\text { Acute ASE0.05 } \\
\text { vs. Acute ASE0.1 } \\
\text { vs. Acute ASE0.3 }\end{array}$} & \multicolumn{2}{|c|}{$\begin{array}{c}\text { Chronic ASE0.05 vs. } \\
\text { Chronic ASE0.1 vs. } \\
\text { Chronic ASE0.3 }\end{array}$} & \multicolumn{2}{|c|}{$\begin{array}{c}\text { Acute ASE0.05 } \\
\text { vs. Chronic ASE0.05 }\end{array}$} & \multicolumn{2}{|c|}{$\begin{array}{l}\text { Acute ASE0.1 } \\
\text { vs. Chronic ASE0.1 }\end{array}$} & \multicolumn{2}{|c|}{$\begin{array}{c}\text { Acute ASE0.3 } \\
\text { vs. Chronic ASE0.3 }\end{array}$} \\
\hline & & $F_{d f}$ & $p$ & $F_{d f}$ & $p$ & $F_{d f}$ & $p$ & $F_{d f}$ & $p$ & $F_{d f}$ & $p$ \\
\hline \multirow{6}{*}{ 菉 } & DM & $0.20_{2,17}$ & $\mathrm{~ns}$ & $0.50_{2,17}$ & $\mathrm{~ns}$ & $6.86_{1,17}$ & 0.018 & $12.14_{1,17}$ & 0.003 & $2.85_{1,17}$ & $\mathrm{~ns}$ \\
\hline & DL & $0.92,17$ & ns & $0.90_{2,17}$ & ns & $5.83_{1,17}$ & 0.027 & $21.07_{1,17}$ & 0.0005 & $13.79_{1,17}$ & 0.002 \\
\hline & VM & $0.22_{2,17}$ & ns & $1.73_{2,17}$ & ns & $11.48_{1,17}$ & 0.003 & $15.81_{1,17}$ & 0.001 & $6.55_{1,17}$ & 0.022 \\
\hline & VL & $069 \cdot 2,17$ & $\mathrm{~ns}$ & $0.49_{2,17}$ & $\mathrm{~ns}$ & $1.88_{1,17}$ & $\mathrm{~ns}$ & $10.17_{1,17}$ & 0.005 & $6.36_{1,17}$ & 0.022 \\
\hline & $\mathrm{CAb}$ & $1.88_{2,17}$ & ns & $0.03_{2,17}$ & $\mathrm{~ns}$ & $2.36_{1,17}$ & ns & $11.32_{1,17}$ & 0.004 & $5.42_{1,17}$ & 0.033 \\
\hline & $\mathrm{SAb}$ & $0.26_{2,17}$ & $\mathrm{~ns}$ & $0.09_{2,17}$ & $\mathrm{~ns}$ & $0.80_{1,17}$ & $\mathrm{~ns}$ & $2.84_{1,17}$ & $\mathrm{~ns}$ & $1.04_{1,17}$ & $\mathrm{~ns}$ \\
\hline \multirow{5}{*}{ 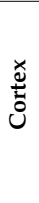 } & ACC & $0.25_{2,17}$ & $\mathrm{~ns}$ & $0.49_{2,17}$ & ns & $1.15_{1,17}$ & ns & $1.20_{1,17}$ & ns & $0.06_{1,17}$ & $\mathrm{~ns}$ \\
\hline & MAC & $0.53_{2,17}$ & ns & $0.61_{2,17}$ & ns & $0.00_{1,17}$ & ns & $3.86_{1,17}$ & ns & $0.05_{1,17}$ & ns \\
\hline & MC & $0.22_{2,17}$ & $\mathrm{~ns}$ & $1.02_{2,17}$ & ns & $1.32_{1,17}$ & $\mathrm{~ns}$ & $8.56_{1,17}$ & 0.009 & $1.00_{1,17}$ & $\mathrm{~ns}$ \\
\hline & SS & $0.24_{2,17}$ & $\mathrm{~ns}$ & $0.75_{2,17}$ & ns & $1.55_{1,17}$ & ns & $7.63_{1,17}$ & 0.013 & $0.86_{1,17}$ & $\mathrm{~ns}$ \\
\hline & IC & $0.29_{2,17}$ & ns & $0.52_{2,17}$ & ns & $1.10_{1,17}$ & ns & $4.46_{1,17}$ & 0.007 & $6.32_{1,17}$ & 0.022 \\
\hline
\end{tabular}

At the multivariate analysis, we found a statistically significant interaction effect between time, asenapine dose, and ROI (Table 4). Significant ROI*time and ROI*asenapine dose interactions were subsequently found, while no asenapine dose*time interaction was observed (Table 4).

\subsubsection{Cortex}

At both the acute and chronic time-points, no significant asenapine dose effect was found in cortical ROIs (Table 1, Figure 2).

A statistically significant effect of time was found in multiple cortical ROIs (Table 1). Homer1a expression was significantly higher after acute compared to chronic administration of ASE0.1 in MC, SS, and IC and of ASE0.3 in IC (Table 1, Figure 2).

At the multivariate analysis, no significant three-way interaction between ROI, asenapine dose, and time was found (Table 4). A significant ROI*time two-way interaction was found, while no significant asenapine dose* $\mathrm{ROI}$ and asenapine dose*time two-way interactions were observed (Table 4).

\subsection{Paradigm 2}

In this paradigm, the three categorical independent variables were: Treatment with incremental haloperidol doses (thereafter haloperidol doses); time; and ROIs. The haloperidol doses variable comprised three categories: $0.25 \mathrm{mg} / \mathrm{kg}$ treatment group; $0.5 \mathrm{mg} / \mathrm{kg}$ treatment group; and $0.8 \mathrm{mg} / \mathrm{kg}$ treatment group. The time variable comprised two categories: Acute administration and chronic administration. The ROIs variable comprised five categories in the cortex (ACC; MAC; MC; SS; IC) and six categories in the striatum (DM; DL; VM; VL; CAb; $\mathrm{SAb}$ ). 


\subsubsection{Striatum}

A significant haloperidol dose effect was observed in the DM and the SAb after acute administration (Table 2, Figure 3). In the DM, Homer1a expression by HAL0.5 was significantly higher than expression by HAL0.8 (Bonferroni adjusted post-hoc test: $p=0.05$; Figure 3). In the SAb, HAL0.5 administrations triggered significantly higher expression of Homer1a than HAL0.25 (Bonferroni adjusted post-hoc test: $p=0.032$; Figure 3). At the chronic time-point, no significant dose effect was found in any ROI (Table 2, Figure 3).

a)

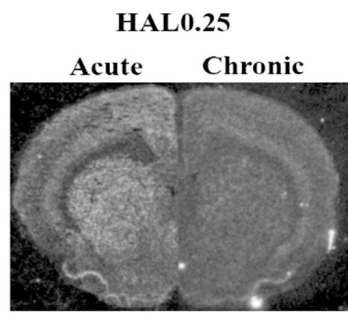

b)
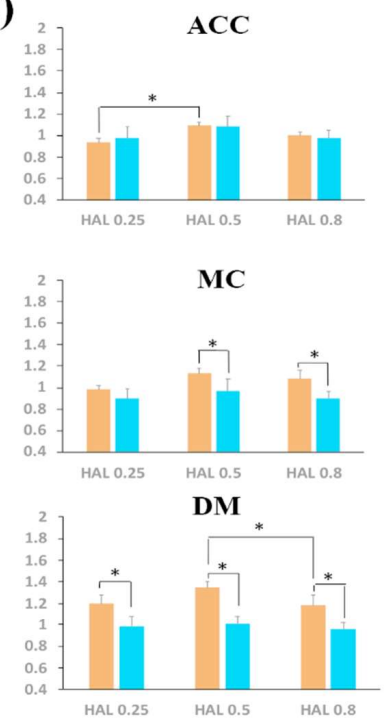

VL

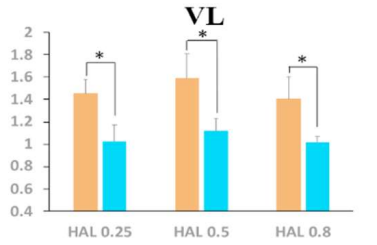

HALO.5
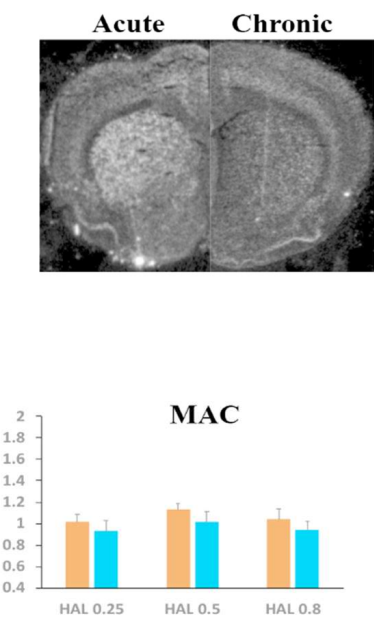

SS
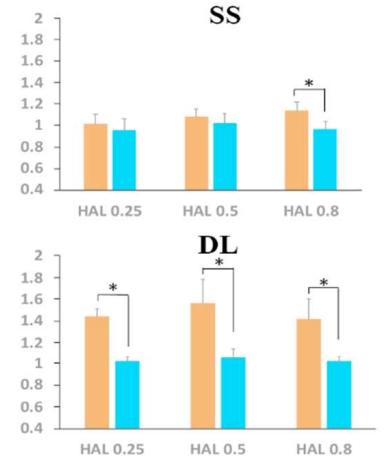

CAb

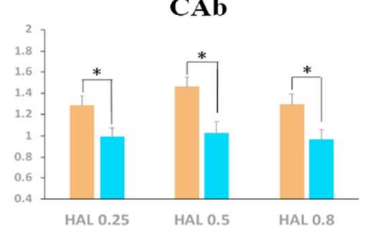

HALO.8

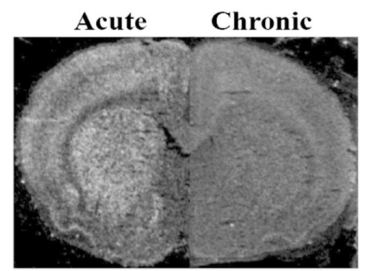

Acute

Chronic

IC
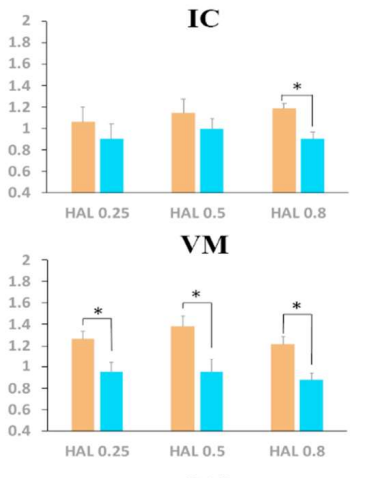

SAb

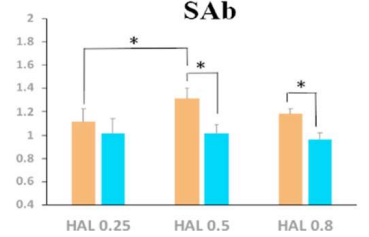

Figure 3. (a) Upper section: representative juxtaposed autoradiogram sections from acute (left side) and chronic (right side) treatment by haloperidol at $0.25 \mathrm{mg} / \mathrm{kg}$ (HAL 0.25), $0.5 \mathrm{mg} / \mathrm{kg}$ (HAL 0.5), and $0.8 \mathrm{mg} / \mathrm{kg}$ (HAL 0.8), respectively; (b) Lower section: levels of Homer1a mRNA expression by different doses of haloperidol at either acute (orange bars) or chronic (turkey bars) time points in the cortical and striatal regions of interest (ROIs). Gene expression is given as fold changes of vehicle-induced relative DPMs \pm standard error mean (S.E.M.). HAL0.25 $=$ haloperidol at $0.25 \mathrm{mg} / \mathrm{kg}$; HAL0.5 = haloperidol at $0.5 \mathrm{mg} / \mathrm{kg}$; HAL0.8 = haloperidol at $0.8 \mathrm{mg} / \mathrm{kg} ;$ ACC = anterior cingulated cortex; $\mathrm{MAC}=$ medial agranular cortex; $\mathrm{MC}=$ motor cortex; $\mathrm{SS}=$ somatosensory cortex; $\mathrm{IC}=$ insular cortex; $\mathrm{DM}=$ dorso-medial caudate putamen; $\mathrm{DL}=$ dorso-lateral caudate putamen; $\mathrm{VM}=$ ventro-medial caudate putamen; $\mathrm{VL}=$ ventro-lateral caudate putamen; $\mathrm{Cab}=$ core of the nucleus accumbens; Sab = shell of the nucleus accumbens; *: significantly different groups at Bonferroni's post-hoc test (haloperidol dose effect) or at Student's t test (time effect). Significantly different groups are connected by brackets. 
Table 2. Dose and time-independent effects on haloperidol-mediated Homer1a expression as assessed by univariate analysis in each region of interest. On the right side of the table, the "dose effect" was analyzed separately for acute and chronic administration regimens, in order to determine if haloperidol-mediated Homer1a expression was different for groups exposed to different haloperidol doses (i.e., HAL0.25, HAL0.5, and HAL0.8). On the left side of the table, the "time effect" was analyzed separately for each haloperidol dose in order to determine if haloperidol-mediated Homer1a expression was different for groups with different durations of treatment (i.e., acute and chronic). Significant effects are given in bold. HAL0.25 = haloperidol at $0.25 \mathrm{mg} / \mathrm{kg}$; HAL0.5 = haloperidol at $0.5 \mathrm{mg} / \mathrm{kg}$; HAL0.8 = haloperidol at $0.8 \mathrm{mg} / \mathrm{kg}$. $\mathrm{ns}=$ not significant.

\begin{tabular}{|c|c|c|c|c|c|c|c|c|c|c|c|}
\hline & & \multicolumn{4}{|c|}{ Dose Effect } & & & \multicolumn{4}{|c|}{ Time Effect } \\
\hline & & \multicolumn{2}{|c|}{$\begin{array}{c}\text { Acute HAL0.25 } \\
\text { vs. Acute HAL0.5 } \\
\text { vs. Acute HAL0.8 }\end{array}$} & \multicolumn{2}{|c|}{$\begin{array}{c}\text { Chronic HAL0.25 } \\
\text { vs. Chronic HAL0.5 } \\
\text { vs. Chronic HAL0.8 }\end{array}$} & \multicolumn{2}{|c|}{$\begin{array}{l}\text { Acute HAL0.25 } \\
\text { vs. Chronic } \\
\text { HAL0.25 }\end{array}$} & \multicolumn{2}{|c|}{$\begin{array}{c}\text { Acute HAL0.5 } \\
\text { vs. Chronic HAL0.5 }\end{array}$} & \multicolumn{2}{|c|}{$\begin{array}{l}\text { Acute HAL0.8 } \\
\text { vs. Chronic HAL0.8 }\end{array}$} \\
\hline & & $F_{d f}$ & $p$ & $F_{d f}$ & $p$ & $F_{d f}$ & $p$ & $F_{d f}$ & $p$ & $F_{d f}$ & $p$ \\
\hline \multirow{6}{*}{ 咅 } & DM & $4.04_{2,16}$ & 0.038 & $0.43_{2,16}$ & ns & $11.40_{1,16}$ & 0.004 & $28.33_{1,16}$ & 0.0005 & $14.60_{1,16}$ & 0.002 \\
\hline & DL & $1.17_{2,16}$ & $\mathrm{~ns}$ & $0.07_{2,16}$ & ns & $16.18_{1,16}$ & 0.001 & $24.50_{1,16}$ & 0.0005 & $17.02_{1,16}$ & 0.001 \\
\hline & VM & $3.37_{2,16}$ & $\mathrm{~ns}$ & $0.88_{2,16}$ & ns & $19.90_{1,16}$ & 0.0005 & $39.80_{1,16}$ & 0.0005 & $26.74_{1,16}$ & 0.0005 \\
\hline & VL & $1.44_{2,16}$ & $\mathrm{~ns}$ & $0.59_{2,16}$ & ns & $13.35_{1,16}$ & 0.002 & $16.47_{1,16}$ & 0.001 & $12.91_{1,16}$ & 0.002 \\
\hline & $\mathrm{CAb}$ & $3.34_{2,16}$ & $\mathrm{~ns}$ & $0.31_{2,16}$ & ns & $14.30_{1,16}$ & 0.002 & $33.84_{1,16}$ & 0.0005 & $22.26_{1,16}$ & 0.0005 \\
\hline & $\mathrm{SAb}$ & $4.20_{2,16}$ & 0.034 & $0.40_{2,16}$ & $\mathrm{~ns}$ & $2.04_{1,16}$ & $\mathrm{~ns}$ & $19.33_{1,16}$ & 0.0005 & $11.89_{1,16}$ & 0.003 \\
\hline \multirow{5}{*}{ 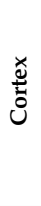 } & $\mathrm{ACC}$ & $4.4_{2,16}$ & 0.03 & $3.08_{2,16}$ & ns & $0.48_{1,16}$ & $\mathrm{~ns}$ & $0.04_{1,16}$ & ns & $0.32_{1,16}$ & $\mathrm{~ns}$ \\
\hline & MAC & $1.6_{2,16}$ & $\mathrm{~ns}$ & $0.87_{2,16}$ & ns & $1.54_{1,16}$ & $\mathrm{~ns}$ & $3.11_{1,16}$ & ns & $2.19_{1,16}$ & $\mathrm{~ns}$ \\
\hline & MC & $3.17_{2,16}$ & ns & $0.74_{2,16}$ & $\mathrm{~ns}$ & $1.76_{1,16}$ & $\mathrm{~ns}$ & $7.59_{1,16}$ & 0.014 & $10.39_{1,16}$ & 0.005 \\
\hline & SS & $2.13_{2,16}$ & $\mathrm{~ns}$ & $0.70_{2,16}$ & $\mathrm{~ns}$ & $0.70_{1,16}$ & $\mathrm{~ns}$ & $0.69_{1,16}$ & $\mathrm{~ns}$ & $8.03_{1,16}$ & 0.021 \\
\hline & IC & $1.39_{2,16}$ & $\mathrm{~ns}$ & $0.82_{2,16}$ & ns & $3.37_{1,16}$ & $\mathrm{~ns}$ & $3.48_{1,16}$ & ns & $13.67_{1,16}$ & 0.002 \\
\hline
\end{tabular}

A significant time effect was found in all striatal ROIs (Table 2). Specifically, in all ROIs, acute expression of Homer1 $a$ was significantly higher than chronic expression by HAL0.25, HAL0.5, and HAL0.8, with the only exception of the SAb for the HAL0.25 dose (Table 2, Figure 3).

At the multivariate analysis, we did not find a significant three-way interaction effect between ROI, haloperidol dose, and time (Table 4). A significant ROI*time two-way interaction was found, while no significant haloperidol dose* $\mathrm{ROI}$ and haloperidol dose*time two-way interactions were observed (Table 4).

\subsubsection{Cortex}

A significant haloperidol dose effect was found in the ACC at the acute time-point (Table 2). In this region, Homer1a expression by HAL0.5 was significantly higher than expression by HAL0.25 (Bonferroni adjusted post-hoc test: $p=0.027$; Figure 3). At the chronic time-point, no significant haloperidol dose effect was found (Table 2, Figure 3).

A significant time effect was found in the MC, SS, and IC regions (Table 2). In all these regions, Homer1a expression by HAL0.8 was significantly higher after the acute than the chronic administration regimen (Figure 3). In the MC, Homer1a expression by acute HAL0.5 was significantly higher than by chronic HAL0.5 administration (Table 2, Figure 3).

At the multivariate analysis, no significant three-way interaction between ROI, haloperidol dose, and time was observed (Table 4). A significant ROI*time two-way interaction was found, while no significant haloperidol dose* $\mathrm{ROI}$ and haloperidol dose*time two-way interactions were observed (Table 4).

\subsection{Paradigm 3}

In this paradigm, the three categorical independent variables were: Treatment with antipsychotics with different receptor profiles (thereafter treatment); time; and ROIs. The treatment variable comprised three categories: Olanzapine $2.5 \mathrm{mg} / \mathrm{kg}$ treatment group; haloperidol $0.5 \mathrm{mg} / \mathrm{kg}$ treatment group; and asenapine $0.1 \mathrm{mg} / \mathrm{kg}$ treatment group. The time variable comprised two categories: 
Acute administration and chronic administration. The ROIs variable comprised five categories in the cortex (ACC; MAC; MC; SS; IC) and six categories in the striatum (DM; DL; VM; VL; CAb; SAb).

The choice of antipsychotic doses to be compared was based on multiple considerations. These doses were reported to elicit a striatal D2 receptor occupancy ranging between $80 \%$ and $90 \%$ [ 42 ], and to exert comparable effects in both behavioral tasks relevant to antipsychotic action $[43,44]$ and studies evaluating neurophysiological and neurochemical outcomes [45-47].

\subsubsection{Striatum}

A significant treatment effect was found in the $\mathrm{DM}, \mathrm{VM}, \mathrm{VL}$, and $\mathrm{CAb}$ regions at the acute time point (Table 3). In all these regions, HAL0.5 triggered significantly higher Homer1a expression than OLA2.5 (Bonferroni adjusted post-hoc test: DM, $p=0.016$; VM, $p=0.014 ; \mathrm{VL}, p=0.031$; CAb, $p=0.013$; Figure 4). No significant treatment effect was observed after chronic antipsychotic administration (Table 3 ).

a)

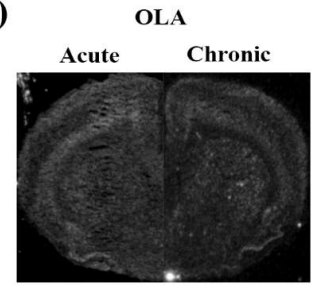

b)
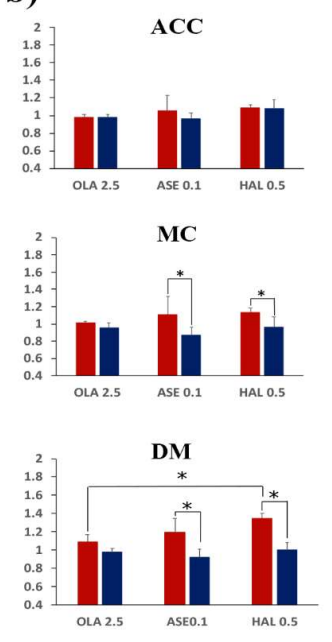

VL

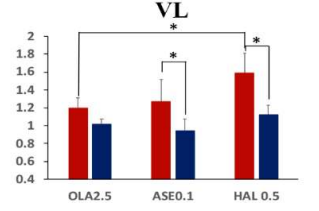

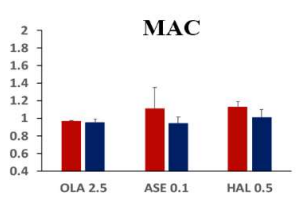
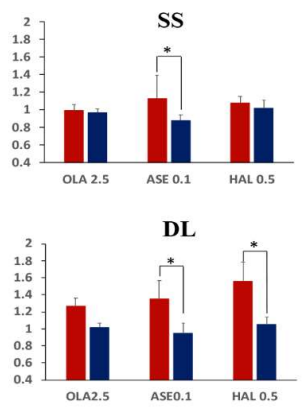

CAb

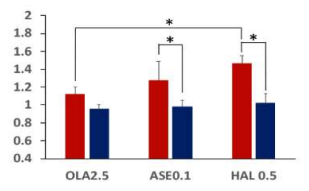

HAL0.5

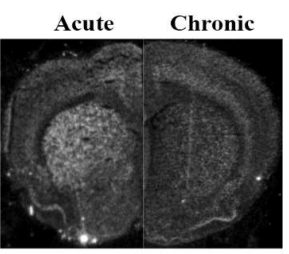

- Acute

- Chronic
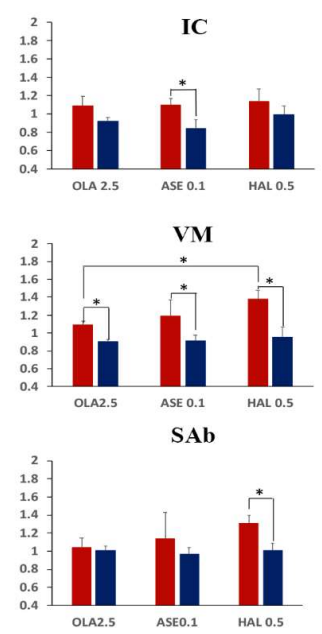

Figure 4. (a) Upper section: representative juxtaposed autoradiogram sections from acute (left side) and chronic (right side) treatment by olanzapine (OLA2.5), asenapine $0.1 \mathrm{mg} / \mathrm{kg}$ (ASE 0.1), and haloperidol $0.5 \mathrm{mg} / \mathrm{kg}$ (HAL 0.5), respectively; (b) Lower section: levels of Homer1a mRNA expression by olanzapine, asenapine, and haloperidol at either acute (red bars) or chronic (dark blue bars) time points in the cortical and striatal ROIs. Gene expression is given as fold changes of vehicle-induced relative DPMs \pm standard error mean (S.E.M.). OLA2.5 = olanzapine at $2.5 \mathrm{mg} / \mathrm{kg}$; ASE0.1 = asenapine at $0.1 \mathrm{mg} / \mathrm{kg}$; HAL0.5 = haloperidol at $0.5 \mathrm{mg} / \mathrm{kg} ; \mathrm{ACC}=$ anterior cingulated cortex; $\mathrm{MAC}=$ medial agranular cortex; $\mathrm{MC}=$ motor cortex; SS = somatosensory cortex; IC = insular cortex; $\mathrm{DM}=$ dorso-medial caudate putamen; $\mathrm{DL}=$ dorso-lateral caudate putamen; $\mathrm{VM}=$ ventro-medial caudate putamen; $\mathrm{VL}=$ ventro-lateral caudate putamen; $\mathrm{Cab}=$ core of the nucleus accumbens; Sab = shell of the nucleus accumbens. *: significantly different groups at Bonferroni's post-hoc test (treatment effect) or at Student's t test (time effect). Significantly different groups are connected by brackets. 
Table 3. Treatment and time-independent effects of different receptor profile antipsychotics on Homer1a expression as assessed by univariate analysis in each region of interest. The treatment effect was analyzed separately for acute and chronic administration regimens. The time effect was analyzed separately for each antipsychotic treatment group (i.e., OLA2.5, ASE0.1, and HAL0.5). Significant effects are given in bold. OLA2.5 = olanzapine at $2.5 \mathrm{mg} / \mathrm{kg}$; ASE0.1 = asenapine at $0.1 \mathrm{mg} / \mathrm{kg} ; \mathrm{HAL0.5}=$ haloperidol at $0.5 \mathrm{mg} / \mathrm{kg} . \mathrm{ns}=$ not significant.

\begin{tabular}{|c|c|c|c|c|c|c|c|c|c|c|c|}
\hline & & \multicolumn{4}{|c|}{ Treatment Effect } & \multicolumn{6}{|c|}{ Time Effect } \\
\hline & & \multicolumn{2}{|c|}{$\begin{array}{l}\text { Acute OLA2.5 vs. } \\
\text { Acute ASE0.1 } \\
\text { vs. Acute HAL0.5 }\end{array}$} & \multicolumn{2}{|c|}{$\begin{array}{c}\text { Chronic OLA2.5 } \\
\text { vs. Chronic ASE0.1 } \\
\text { vs. Chronic HAL0.5 }\end{array}$} & \multicolumn{2}{|c|}{$\begin{array}{l}\text { Acute OLA2.5 } \\
\text { vs. Chronic OLA2.5 }\end{array}$} & \multicolumn{2}{|c|}{$\begin{array}{c}\text { Acute ASE0.1 } \\
\text { vs. Chronic ASE0.1 }\end{array}$} & \multicolumn{2}{|c|}{$\begin{array}{l}\text { Acute HAL } 0.5 \text { vs. } \\
\text { Chronic HAL0.5 }\end{array}$} \\
\hline & & $F_{d f}$ & $p$ & $F_{d f}$ & $p$ & $F_{d f}$ & $p$ & $F_{d f}$ & $p$ & $F_{d f}$ & $p$ \\
\hline \multirow{6}{*}{ 咅 } & DM & $5.45_{2,15}$ & 0.017 & $0.76_{2,15}$ & $\mathrm{~ns}$ & $1.96_{1,15}$ & ns & $12.14_{1,17}$ & 0.003 & $28.33_{1,16}$ & 0.0005 \\
\hline & DL & $3.00_{2,15}$ & ns & $0.50_{2,15}$ & $\mathrm{~ns}$ & $3.86_{1,15}$ & ns & $21.07_{1,17}$ & 0.0005 & $24.50_{1,16}$ & 0.0005 \\
\hline & VM & $5.75_{2,15}$ & 0.014 & $0.20_{2,15}$ & ns & $4.47_{1,15}$ & 0.05 & $15.81_{1,17}$ & 0.001 & $39.80_{1,16}$ & 0.0005 \\
\hline & VL & $4.91_{2,15}$ & 0.023 & $1.15_{2,15}$ & $\mathrm{~ns}$ & $1.68_{1,15}$ & ns & $10.17_{1,17}$ & 0.005 & $16.47_{1,16}$ & 0.001 \\
\hline & $\mathrm{CAb}$ & $5.65_{2,15}$ & 0.015 & $0.25_{2,15}$ & $\mathrm{~ns}$ & $2.86_{1,15}$ & ns & $11.32_{1,17}$ & 0.004 & $33.84_{1,16}$ & 0.0005 \\
\hline & $\mathrm{SAb}$ & $2.23_{2,15}$ & $\mathrm{~ns}$ & $0.18_{2,15}$ & $\mathrm{~ns}$ & $0.05_{1,15}$ & ns & $2.84_{1,17}$ & ns & $19.33_{1,16}$ & 0.0005 \\
\hline \multirow{5}{*}{ 离 } & ACC & $0.99_{2,15}$ & ns & $1.57_{2,15}$ & $\mathrm{~ns}$ & $0.00_{1,15}$ & ns & $1.20_{1,17}$ & ns & $0.04_{1,16}$ & ns \\
\hline & MAC & $1.55_{2,15}$ & ns & $0.36_{2,15}$ & ns & $0.02_{1,15}$ & ns & $3.86_{1,17}$ & ns & $3.11_{1,16}$ & ns \\
\hline & MC & $0.90_{2,15}$ & ns & $0.69_{2,15}$ & ns & $0.42_{1,15}$ & ns & $8.56_{1,17}$ & 0.009 & $7.59_{1,16}$ & 0.014 \\
\hline & SS & $0.84_{2,15}$ & ns & $1.20_{2,15}$ & $\mathrm{~ns}$ & $0.05_{1,15}$ & $\mathrm{~ns}$ & $7.63_{1,17}$ & 0.013 & $0.69_{1,16}$ & ns \\
\hline & IC & $0.15_{2,15}$ & ns & $1.25_{2,15}$ & ns & $2.68_{1,15}$ & ns & $4.46_{1,17}$ & 0.007 & $3.48_{1,16}$ & ns \\
\hline
\end{tabular}

A significant time effect was found in all striatal ROIs (Table 3). Acute HAL0.5 and ASE0.1 induced higher Homer1a expression than chronic HAL0.5 and ASE0.1, respectively, in all striatal regions, with the exception of the SAb for ASE0.1 (Figure 4). Acute OLA2.5 triggered higher Homer1a expression than chronic OLA2.5 in the VM (Figure 4).

At the multivariate analysis, no significant three-way interaction between ROI, treatment, and time was observed (Table 4). A significant ROI*time two-way interaction was found, while no significant treatment*ROI and treatment*time two-way interactions were observed (Table 4).

Table 4. Overall output of multivariate statistical analyses. In this table, we report the overall effects of multivariate statistical analyses carried out in the present study. Three-way interaction reported the effect of $\mathrm{ROI}^{*}$ dose/treatment*time. Two-way effects were subdivided in within subjects (i.e., $\mathrm{ROI}^{*}$ dose/treatment and $\mathrm{ROI}^{*}$ time) and between subjects (i.e., dose/treatment ${ }^{*}$ time). Bold highlight indicates significant effect. $\mathrm{ROI}=$ Regions of Interest; $\mathrm{ASE}=$ asenapine; $\mathrm{HAL}=$ haloperidol; DRPA $=$ different receptor profile antipsychotics.

\begin{tabular}{|c|c|c|c|c|}
\hline & $\begin{array}{l}\text { Three-Way } \\
\text { Interaction }\end{array}$ & $\begin{array}{c}\text { Two-Way } \\
\text { Within-Subject } \\
\text { (ROI*Dose/Treatment) }\end{array}$ & $\begin{array}{c}\text { Two-Way } \\
\text { Within-Subject } \\
\text { (ROI*Time) }\end{array}$ & $\begin{array}{c}\text { Two-Way } \\
\text { Between-Subject } \\
\text { (Dose/Treatment*Time) }\end{array}$ \\
\hline ASE Striatum & $\begin{array}{c}F_{10,85}=1.99 \\
P=0.04\end{array}$ & $\begin{array}{c}F_{10,85}=2.46 \\
P=0.012\end{array}$ & $\begin{array}{c}F_{5,85}=11.66 \\
P<0.0005\end{array}$ & $\begin{array}{c}\mathrm{F}_{2,17}=0.53 \\
P>0.05\end{array}$ \\
\hline $\begin{array}{c}\text { ASE } \\
\text { Cortex }\end{array}$ & $\begin{array}{c}\mathrm{F}_{8,68}=1.31 \\
P>0.05\end{array}$ & $\begin{array}{c}\mathrm{F}_{8,68}=0.29 \\
P>0.05\end{array}$ & $\begin{array}{c}F_{4,68}=12.42 \\
P<0.0005\end{array}$ & $\begin{array}{c}\mathrm{F}_{2,17}=0.81 \\
P>0.05\end{array}$ \\
\hline HAL Striatum & $\begin{array}{c}\mathrm{F}_{10,80}=0.51 \\
P>0.05\end{array}$ & $\begin{array}{c}\mathrm{F}_{10,80}=0.57 \\
P>0.05\end{array}$ & $\begin{array}{c}F_{5,80}=12.74 \\
P<0.0005\end{array}$ & $\begin{array}{c}\mathrm{F}_{2,16}=0.81 \\
P>0.05\end{array}$ \\
\hline $\begin{array}{c}\text { HAL } \\
\text { Cortex }\end{array}$ & $\begin{array}{c}\mathrm{F}_{8,64}=1.23 \\
P>0.05\end{array}$ & $\begin{array}{c}\mathrm{F}_{8,64}=1.52 \\
P>0.05\end{array}$ & $\begin{array}{c}F_{4,64}=13.02 \\
P<0.0005\end{array}$ & $\begin{array}{c}\mathrm{F}_{2,16}=0.57 \\
P>0.05\end{array}$ \\
\hline DRPA Striatum & $\begin{array}{c}\mathrm{F}_{10,75}=0.19 \\
P>0.05\end{array}$ & $\begin{array}{c}\mathrm{F}_{10,75}=1.44 \\
P>0.05\end{array}$ & $\begin{array}{c}F_{5,75}=8.64 \\
P<0.0005\end{array}$ & $\begin{array}{c}\mathrm{F}_{2,15}=1.83 \\
P>0.05\end{array}$ \\
\hline DRPA Cortex & $\begin{array}{c}\mathrm{F}_{8,60}=1.31 \\
P>0.05\end{array}$ & $\begin{array}{c}\mathrm{F}_{8,60}=1.19 \\
P>0.05\end{array}$ & $\begin{array}{l}F_{4,60}=9.17 \\
P<0.0005\end{array}$ & $\begin{array}{c}\mathrm{F}_{2,15}=0.48 \\
P>0.05\end{array}$ \\
\hline
\end{tabular}

\subsubsection{Cortex}

No significant treatment effect was observed at either the acute or the chronic time-point (Table 3). 
A significant time effect was found in the MC, SS, and IC regions (Table 3). In all these regions, ASE0.1 acute administration induced significantly higher Homer1a expression than ASE0.1 chronic administration (Figure 4). In the MC, acute HAL0.5 also induced significantly higher Homer1a expression than chronic HAL0.5 (Figure 4).

At the multivariate analysis, no significant three-way interaction between ROI, treatment, and time was observed (Table 4). A significant ROI*time two-way interaction was found, while no significant treatment*ROI and treatment*time two-way interactions were observed (Table 4).

\section{Discussion}

In this work, we aimed to evaluate whether the molecular effects exerted by antipsychotics on the Homer1a gene, which is a marker of glutamatergic synaptic plasticity and dopamine-glutamate interaction, were modulated by the antipsychotic dose/receptor profile, the duration of administration (acute vs. repeated), and brain regions evaluated. A graphical summarization of the major results of this study is given in Figures 5 and 6.
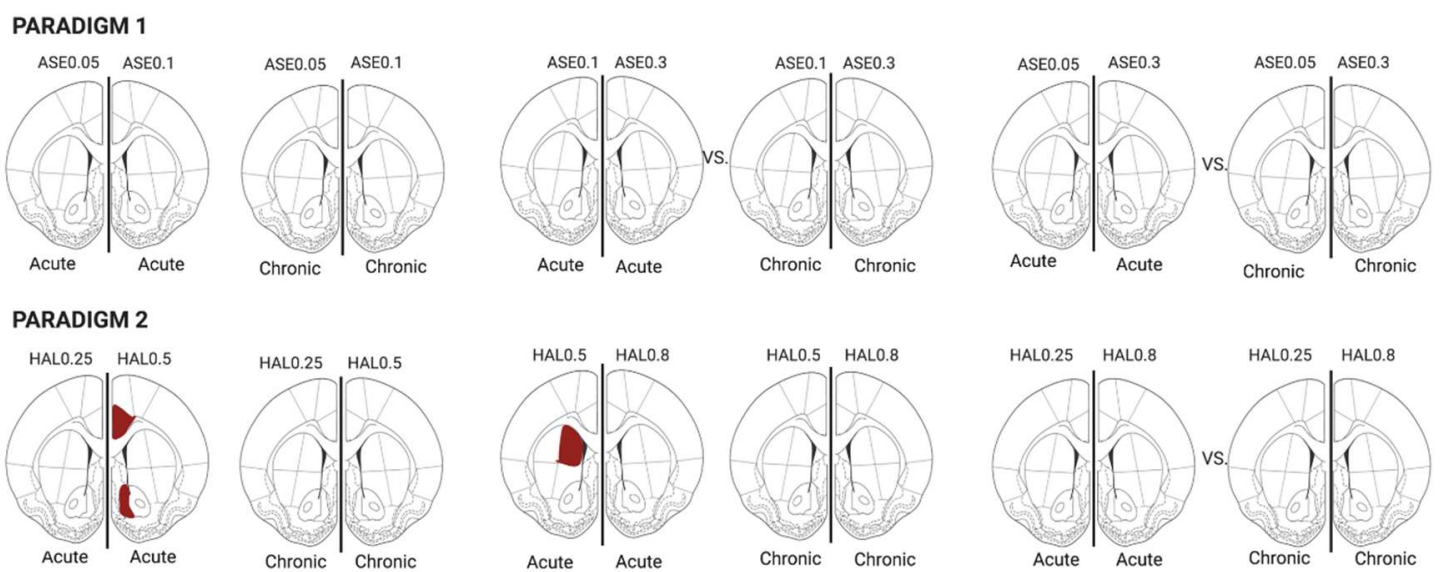

\section{PARADIGM 3}
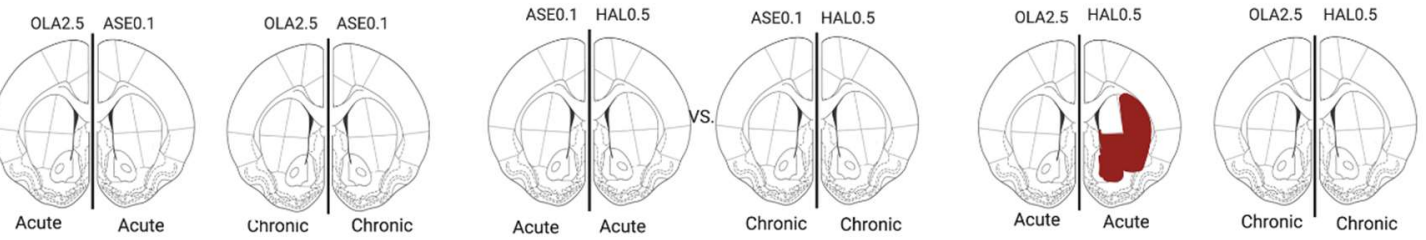

Figure 5. The treatment effect was analyzed for each region of interest (ROI) at both acute and chronic time-points. The red-colored ROI are those expressing significantly higher Homer1a levels compared to the corresponding ROI at the other treatment regimen. $\mathrm{VEH}=$ vehicle; ASE0.05 = asenapine $0.05 \mathrm{mg} / \mathrm{kg}$; ASE0.1 = asenapine $0.1 \mathrm{mg} / \mathrm{kg}$; ASE0.3 = asenapine $0.3 \mathrm{mg} / \mathrm{kg} ;$ HAL0.25= haloperidol $0.25 \mathrm{mg} / \mathrm{kg}$; HAL0.5 = haloperidol $0.5 \mathrm{mg} / \mathrm{kg}$; HAL0.8 = haloperidol $0.8 \mathrm{mg} / \mathrm{kg}$; OLA2.5 = olanzapine $2.5 \mathrm{mg} / \mathrm{kg}$.

Among the major findings of this work, we found: (i) Significant treatment effects, since Homer1a expression was different in the selected areas and time-points among antipsychotics with different receptor profiles; (ii) significant dose effects for haloperidol in selected striatal and cortical ROIs at the acute but not the chronic time-point; (iii) significant time effects since Homer1a expression by all antipsychotics was significantly higher after acute administration compared to a prolonged treatment regimen; and (iv) a significant three-way interaction between topography (i.e., ROIs), antipsychotic dose, and time for asenapine in the striatum, and a significant two-way interaction between ROI and time in all groups investigated.

These data indicate that the effects of the duration of antipsychotic treatment on gene expression were affected by the brain regions where gene expression was measured. Moreover, the extent of gene expression, at least by the antipsychotics tested herein, was strongly affected by the duration 
of treatment, with acute (i.e., single administration) regimens inducing significantly higher Homer1a expression than chronic ones (i.e., 21-day-long administration period).

\section{PARADIGM 1}
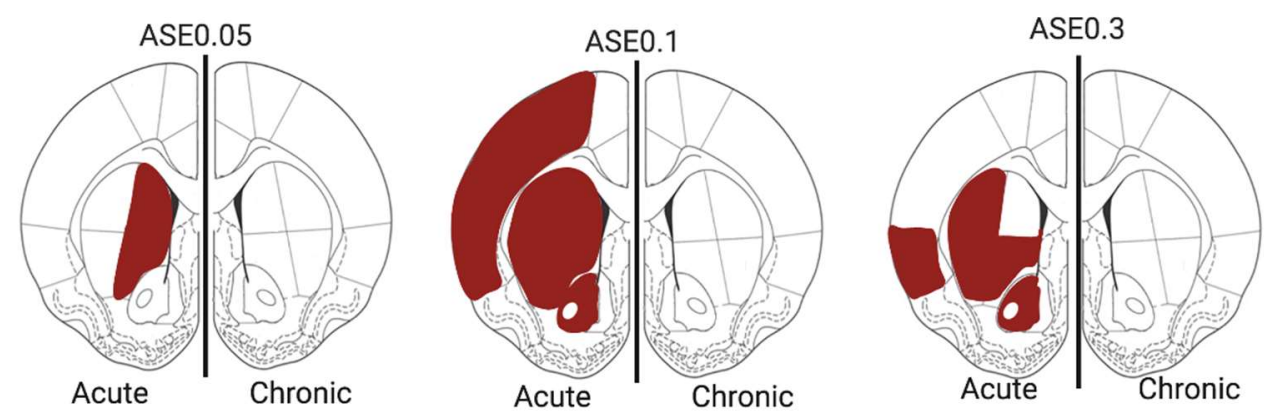

PARADIGM 2
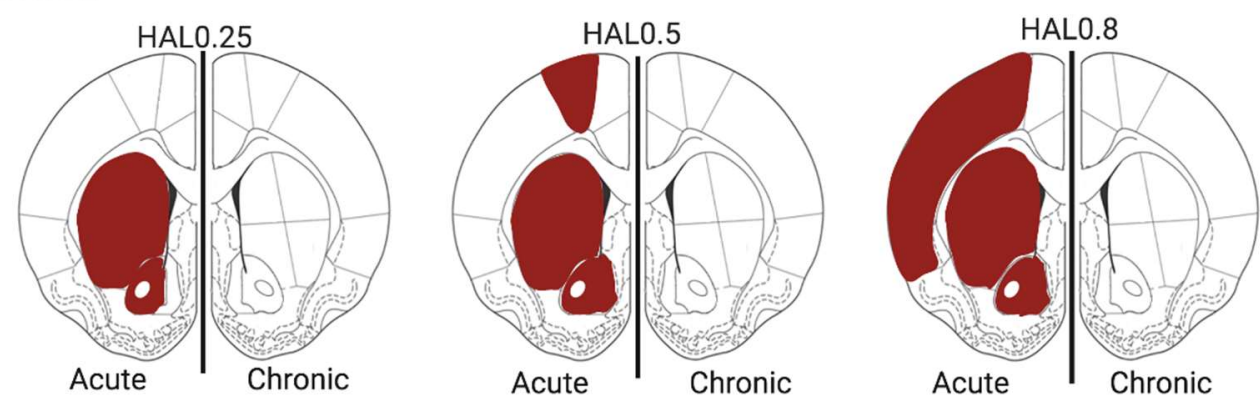

PARADIGM 3
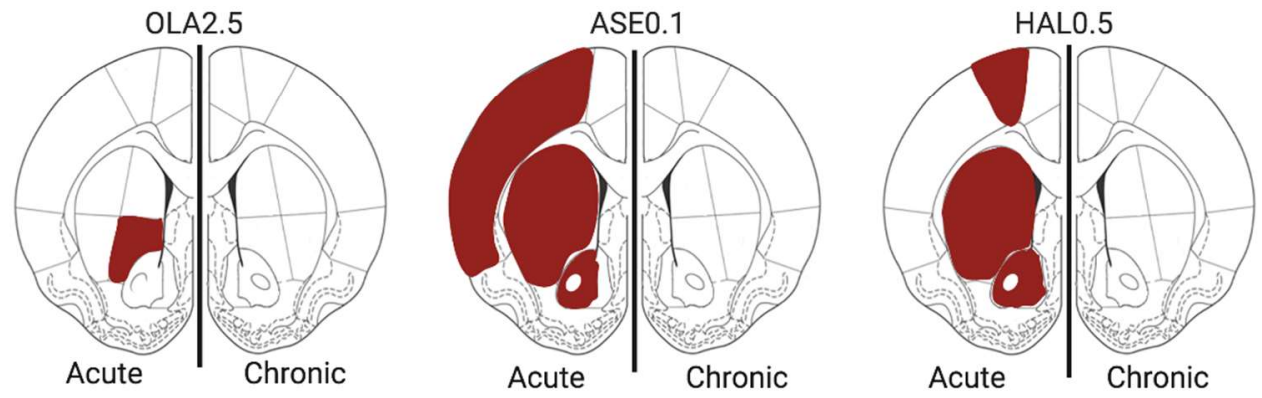

Figure 6. The time effect was analyzed separately for each antipsychotic treatment. The red-colored regions of interest (ROI) were those expressing significantly higher levels of Homer1a in comparison to the corresponding ROI at the other time-point. $\mathrm{VEH}=$ vehicle; ASE0.05 = asenapine $0.05 \mathrm{mg} / \mathrm{kg}$; ASE0.1 = asenapine $0.1 \mathrm{mg} / \mathrm{kg}$; ASE0.3 = asenapine $0.3 \mathrm{mg} / \mathrm{kg} ;$ HAL0.25 = haloperidol $0.25 \mathrm{mg} / \mathrm{kg}$; HAL0.5 = haloperidol $0.5 \mathrm{mg} / \mathrm{kg}$; HAL0.8 = haloperidol $0.8 \mathrm{mg} / \mathrm{kg}$; OLA2.5 = olanzapine $2.5 \mathrm{mg} / \mathrm{kg}$.

The data reinforced the suggestion that the biological effects of antipsychotics on Homer1a depend on a complex combination of the receptor profile, topography, and duration of exposure to these agents. Since Homer is a family of molecules that are crucially implicated in glutamatergic signaling, PSD architecture, and whose expression is profoundly affected by dopaminergic modulation, the evaluation of the present results may take into consideration these biological elements. Antipsychotic-mediated modulation of dopaminergic and possibly non-dopaminergic (including glutamatergic) receptors should be accounted for in mechanistic explanations of changes in Homer1a expression. Homer effects on glutamatergic signaling and synaptic rearrangements should be considered when discussing the antipsychotic-mediated modulation of Homer.

As expected, the first-generation antipsychotic haloperidol induced higher Homer1a expression than olanzapine at the acute time-point in striatal regions. This result is consistent with the hypothesis 
that antipsychotic-induced Homer1a expression in the striatum mainly depends on the degree of the dopamine D2R antagonism, whereby acute administration of potent D2R-blockers induces greater Homer1a expression in comparison to atypical agents in subcortical regions. Hence, acute variations in subcortical Homer1a mRNA have been proposed by our group as predictors of the typical or atypical character of the antipsychotic molecules $[10,48]$. These observations comply with previous reports $[11,20,49,50]$. From a clinical point of view, it may reflect the propensity of typical compounds to provoke extrapyramidal symptoms in humans, as discussed below in more detail.

It has been reported that Homer1a mRNA is under a prominent glutamatergic regulation since NMDAR activation upregulates gene expression [51]. Moreover, Homer1a expression is mediated by selective D2R antagonism [10], and possibly 5-HT2R antagonism and D1R agonism [52,53], either directly or indirectly, via glutamate-mediated pathways. Acute antipsychotic administration is known to increase, rather than decrease, extracellular levels of dopamine in the striatum, exerting a relevant blockade of presynaptic D2Rs [54,55]. Since post-synaptic D2Rs are blocked by the antipsychotic, it may be expected that dopamine may interact with post-synaptic D1Rs. In turn, activated D1Rs may cooperate with NMDARs to intensify NMDAR-mediated glutamatergic transmission [56], leading to increased Homer1a expression. According to these mechanistic considerations, the higher acute Homer1a expression by haloperidol compared to olanzapine appears to indicate a larger action on D2R by haloperidol than olanzapine, which in turn may be responsible for larger presynaptic dopamine release and larger NMDA receptor activation via post-synaptic D1Rs. Although no significant differences between haloperidol and asenapine treatments were found in this study, this may depend on the highly conservative type of post-hoc test we adopted herein, i.e., the Bonferroni correction. Notably, in a previous study [3], we found significant differences between these two agents, corroborating the view above.

A dose effect was found by haloperidol but not by asenapine. Moreover, the dose effect was demonstrated only in selected cortical and striatal areas and only after an acute treatment. Since increased Homer1a expression has been consistently associated to enhanced glutamatergic excitatory activity, possibly as a feedback mechanism to prevent NMDAR-mediated neuronal injury [57-59], our results may indicate that, in selected brain regions, acute haloperidol may trigger higher activation of glutamatergic neurons at the intermediate $0.5-\mathrm{mg}$ compared to the high $0.8-\mathrm{mg}$ dose.

At the 0.8-mg high dose, haloperidol may over-saturate synaptic D2Rs and interact with a fraction of D1Rs as well, competing with synaptic dopamine and therefore partially blocking intracellular transduction from these receptors. Antagonism at D1Rs would, in turn, trigger less NMDAR activation as compared to the activation given by the $0.5-\mathrm{mg}$ dose. As a consequence of this reduced NMDAR activation, less Homer1a mRNA would be produced by neurons compared to the intermediate 0.5-mg dose. Indeed, it has been described that more than $90 \%$ striatal D2Rs are blocked by haloperidol at doses comparable to those used herein $[60,61]$ and that haloperidol has some affinity to D1Rs, although lower than to D2Rs [62].

The loss of this pattern in chronic treatments suggests that these dynamics may be restricted to acute treatments, while more complex molecular rearrangements may occur during sustained antipsychotics, as it will be discussed below. Repeated administration may allow reaching plasma steady-state and targeting high-level D2Rs, thereby smoothing differences among doses. It has been described, by in vivo recordings of striatal D2R occupancy, that 7-day treatment with 0.25 or $0.5 \mathrm{mg} / \mathrm{kg}$ haloperidol resulted in non-significant differences in D2R occupancy, which was higher than $65 \%$ [60]. Additionally, behavioral outcomes, such as vacuous chewing movements, were found to be non-significantly different among haloperidol doses reminiscent of those used herein given for eight weeks [63]. Therefore, these studies support the view that the effects of divergent haloperidol doses mitigate during repeated administration and agree with the molecular observations in the present study. 
The lack of dose dependence by asenapine may be due to the almost equivalent affinity to D1Rs and D2S by this agent or its strong serotonergic action [36,37], which is predicted to affect dopamine release and may prevent the saturation of D2Rs.

It could not be excluded that other receptor systems may intervene in these dynamics, e.g., the sigma1 receptor for haloperidol or the histaminergic, cholinergic, or serotonergic receptors for asenapine. More studies are needed to understand whether this different pattern is specific to relatively selective D2Rs blockers, such as haloperidol, and are avoided in antipsychotics with a broader receptor profile.

A consistent time effect was found for all antipsychotics tested. Accordingly, the biological effects exerted by haloperidol, asenapine, and olanzapine on Homer1a were significantly higher after a single acute administration than after repeated dosing. These results may indicate that antipsychotic effects on the Homer1a-mediated biological system diminish over repeated administrations, although these outcomes vary depending on the antipsychotic dose and brain region.

One possible explanation for these results is that prolonged antipsychotic treatment may be associated with a reduction of the depolarizing effects on neurons. Indeed, it has been demonstrated that prolonged antipsychotic treatments may cause depolarization blocks and reduce synaptic dopamine [64]. This experimental evidence may explain the loss of the dose effect observed in the present study at the chronic treatment.

Another possible explanation is an upregulation of post-synaptic D2Rs, which may cause the behavioral phenomena of sensitization/tolerance to prolonged antipsychotics [65]. The observed attenuation of Homer1a expression after chronic antipsychotics may represent an adaptive biological mechanism predisposing to antipsychotic sensitization/tolerance. Attenuated Homer1a expression may reflect (or cause) a reduction of glutamatergic neurons' activity in targeted areas as compared to acute administration. In turn, this may depend on D2R-related (and possibly 5-HT2A receptor-related) adaptive changes, i.e., upregulation [66,67], since it has been reported that increased D2R activation reduces glutamate activation via NMDA receptors [68].

Indeed, a time effect has been reported in a behavioral task (conditioned avoidance response, CAR) after repeated haloperidol, risperidone, and olanzapine treatments [69]. Specifically, antipsychotic efficacy in suppressing avoidance response had an early onset and increased progressively [69]. On the other hand, our data showed that antipsychotic effects on Homer1a had an early onset but significantly declined over time. Whether these molecular changes may underlie the reported behavioral sensitization effects of antipsychotics in rodents is still challenging.

Notably, Homer1a overexpression in striatal sites has been associated with reduced locomotor activity [70]. Remarkably, glutamatergic hyperactivity in the striatum has been considered a major pathophysiological mechanism in Parkinson's disease as well as in L-DOPA-induced dyskinesia [71-73]. It may be hypothesized that increased Homerla expression by acute antipsychotics may be the consequence of glutamatergic activation in the striatum, which in turn may concur to cause acute extrapyramidal effects. The relative attenuation of Homer $1 a$ expression by persistent antipsychotic treatment may indicate a reduced striatal glutamatergic activation, possibly as a consequence of dopamine receptor sensitization, which may lead to an attenuation of the imbalance in motor-related circuits and reduced motor side effects. This hypothesis paves the way to the possibility of manipulating Homer1a expression as a tool to affect motor side effects of antipsychotics. Nonetheless, this hypothesis should be tested in specifically designed experiments.

Significant acute vs. chronic changes were found in the insular cortex with haloperidol and asenapine. From a neurobiological and neuropharmacological point of view, these results may implicate that the insular cortex represents a preferential site in which antipsychotics exert time-dependent effects, irrespective of the antipsychotic used. From a clinical point of view, the insular cortex has been considered a crucial region for the salience network, which is widely affected by dopaminergic and glutamatergic neurotransmission, and is involved in the ability to discriminate between self-generated and external information [74,75]. Alterations in the salience network and insula overactivations may 
underlie many of the clinical features of psychotic disorders [76,77]. Moreover, antipsychotic treatments have been found to restore insular abnormalities in patterns of connectivity [78]. In this context, decreased Homer1a expression after chronic antipsychotic exposure in this peculiar region may possibly indicate a reduced glutamatergic activation exerted by neuroleptics, which in turn may underpin potential mitigating effects on abnormal salience signaling.

One challenging result of our study is that despite evidence that topography, treatment, and duration of antipsychotic administration may combine to determine multiple differential patterns of Homer1a expression, multivariate analysis of the interactions among these factors was often negative. Despite the fact that the study was adequately powered to run univariate analyses, the power for multivariate analyses was lower, determining the possibility of false negative outcomes.

In conclusion, Homer1a gene expression is affected by antipsychotics with a specific dose, receptor profile, topography, and time-related dynamics. These data may deepen the knowledge of antipsychotics neurobiology and may have translational valence to infer their unique clinical action and side effects.

\section{Materials and Methods}

\subsection{Animals and Drug Treatments}

Male Sprague-Dawley rats, mean weight $250 \mathrm{~g}$ (Charles River Labs, Lecco, Italy), were obtained, and housed in a colony room at controlled temperature and humidity, under a $12 \mathrm{~h}$ light/dark cycle, with ad libitum access to laboratory chow and water. In the first days, animals were adapted to human handling. All procedures were approved by the local Animal Care and Use Committee and were in accordance to the NIH Guide for Care and Use of Laboratory Animals (NIH Publication No. 85-23, revised 1996). We used the least number of animals possible to obtain reliable results.

\subsection{Study Design and Drug Treatment}

The following agents were used: (1) Asenapine maleate (supplied as a powder by H. Lundbeck A/S, Copenhagen, Denmark), olanzapine, and haloperidol (both supplied as a powder by Sigma-Aldrich, Milan, Italy). The powder was dissolved in saline solution ( $\mathrm{NaCl} 0.9 \%)$, adjusted to physiological $\mathrm{pH}$, and injected i.p. (final volume: $1 \mathrm{~mL} / \mathrm{kg}$ ).

A total of 80 animals were used for the experimental procedures. Rats were randomly assigned to one of the following treatments $(n=10$ for each): Vehicle $(\mathrm{NaCl} 0.9 \%, \mathrm{VEH})$; asenapine $0.05 \mathrm{mg} / \mathrm{kg}$ (ASE0.05); asenapine $0.1 \mathrm{mg} / \mathrm{kg}$ (ASE0.1); asenapine $0.3 \mathrm{mg} / \mathrm{kg}$ (ASE0.3); haloperidol $0.25 \mathrm{mg} / \mathrm{kg}$ (HAL0.25); haloperidol $0.5 \mathrm{mg} / \mathrm{kg}$ (HAL0.5); haloperidol $0.8 \mathrm{mg} / \mathrm{kg}$ (HAL0.8); and olanzapine $2.5 \mathrm{mg} / \mathrm{kg}$ (OLA2.5). All drugs were administered at doses that were reported to be behaviorally active in previous works $[3,12,35,38,49,79,80]$. The doses of asenapine and haloperidol were in the dose range that has been used in multiple experimental paradigms to mimic the neurochemical and behavioral effects of antipsychotics [42,47,61,81-84].

Rats were then split into two sets: Those sacrificed $90 \mathrm{~min}$ after single drug injection (i.e., acute dose regimen), and those sacrificed $90 \mathrm{~min}$ after the last administration of a chronic dose regimen (i.e., 21-day consecutive treatment). As a result, rats were ultimately subdivided in the following groups ( $n=5$ each): Acute VEH; chronic VEH; acute ASE0.05; chronic ASE0.05; acute ASE0.1; chronic ASE0.1; acute ASE0.3; chronic ASE0.3; acute HAL0.25; chronic HAL0.25; acute HAL0.5; chronic HAL0.5; acute HAL0.8; chronic HAL0.8; acute OLA2.5; and chronic OLA2.5.

\subsection{In Situ Hybridization}

After sacrifice, animal brains were frozen on powdered dry ice and stored at $-70^{\circ} \mathrm{C}$. Sectioning was made on a cryostat at $-18{ }^{\circ} \mathrm{C}$. Serial coronal sections of $12 \mu \mathrm{m}$ were obtained at the level of the middle-rostral striatum (approximately from Bregma 1.20 to $1.00 \mathrm{~mm}$ ). The rat brain atlas by 
Paxinos and Watson [85] was used as an anatomical reference. Sections were stored at $-80{ }^{\circ} \mathrm{C}$ for subsequent analysis.

We initially designed a series of probes for radioactive in situ hybridization by Gene-Bank, among those oligodeoxyribonucleotide sequences that were found complementary to the mRNA sequence of the Homer1a gene. The initially designed sequences were double-checked with BLAST in order to avoid cross-hybridization. The selected probe was that ensuring the best specificity/sensitivity ratio and was manufactured by MWG Biotech, Milan, Italy.

On the day of the hybridization, the sections were prepared to receive the hybridization mix by sequential washes into $4 \%$ formaldehyde, PBS (pH 7.4), $0.25 \%$ acetic anhydride in $0.1 \mathrm{M}$ triethanolamine $/ 0.9 \% 25 \mathrm{NaCl}$, ethanol, chloroform, again in ethanol, and finally air-dried.

The labeling reaction mix consisted of $100 \mathrm{mCi}{ }^{35} \mathrm{~S}-\mathrm{dATP}, 125$ units of terminal deoxynucleotidyl transferase (TdT), and $7.5 \mathrm{pmol} / \mu \mathrm{L}$ of oligodeoxyribonucleotide. The ProbeQuant $\mathrm{G}-50$ Micro Columns (supplied by Amersham-GE Healthcare Biosciences, Italy) were used to separate unincorporated nucleotides from radiolabeled DNA.

Sections were hybridized with $0.4-0.6 \times 10^{6} 2 \mathrm{cpm}$ of labeling reaction mix that was buffered by $0.2 \mathrm{mg} / \mathrm{mL}$ heparin sulfate, $50 \%$ formamide, $10 \%$ dextran sulfate, $4 \mathrm{mM}$ EDTA, $0.1 \%$ pyrophosphate, $600 \mathrm{mM} \mathrm{NaCl}$, and $80 \mathrm{mM}$ Tris- $\mathrm{HCl}$ (pH 7.5).

Hybridized sections were incubated at $37^{\circ} \mathrm{C}$ in a humidified chamber for $22-24 \mathrm{~h}$ and subsequently washed in $2 \times \mathrm{SSC} / 50 \%$ formamide at $43-44{ }^{\circ} \mathrm{C}$ in $1 \times$ SSC at room temperature. After drying, sections were exposed to an autoradiographic film (Kodak-10 Biomax MR). Sections were co-exposed with a ${ }^{14} \mathrm{C}$ standards' slide (ARC-146C, American Radiolabeled Chemical). Time of exposure was set to maximize the signal-to-noise ratio.

The ImageJ software (v. 1.46v, http://rsb.info.nih.gov/ij/) was used for quantitation of the optical density of the autoradiographic signal. Brain sections from both rats treated acutely and rats treated chronically were co-exposed to the same X-ray sheet to allow comparative statistical analysis. Optical density was measured within outlined regions of interest (ROIs), in correspondence of the cortex (ACC, MAC, MC, SS, and IC), and the striatum (DM, DL, VM, VL, Cab, and Sab) (Figure 1). The ${ }^{14} \mathrm{C}$ standard values from 4 through 12 were previously cross-calibrated to ${ }^{35} \mathrm{~S}$ brain paste standards to obtain a calibration curve based on a "best fit" 3rd-degree polynomial. The calibration curve was used to convert the measurements of the optical density into "relative DPM".

For statistical analysis, data from each antipsychotic treatment were given as fold changes of the gene expression to the vehicle-induced expression. Specifically, acute antipsychotic signal intensity was given as fold changes of the acute vehicle signal intensity, and chronic antipsychotic signal intensity as fold changes of the chronic vehicle. The whole in situ hybridization procedure was performed blinded with coded frozen brains.

\subsection{Statistical Analysis}

The statistical analyses were performed using JMP9.0.1 and SPSS 24.0 software. The Shapiro-Wilk test was used to confirm normal distribution of the continuous variable (i.e., signal intensity of gene expression). ROIs, treatment, and time were the categorical variables that were used to analyze gene expression.

As a first goal, we wanted to capture significant effects of independent time or treatment in any topographic subdivision. Therefore, we carried out separate univariate analyses of time and treatment effect in each ROI. In all tests, significance was set at $p<0.05$. The Bonferroni corrected post-hoc tests were used for pairwise comparisons in univariate analyses.

To contemporarily evaluate the effects of antipsychotic dose/receptor profile, topography (i.e., ROIs), and duration of treatment (i.e., time), we carried out a 3 way mixed ANOVA, with ROIs as the within-subjects factor, and treatment and time as the between-subjects factors. To avoid multiple significant $p$-values of limited practical relevance, cortical and striatal ROIs were separately assessed since they represent distinct anatomical divisions within the forebrain, although functionally connected. 
Follow-up evaluations of 2 way interactions with the within-subjects factor (i.e., $\mathrm{ROI}^{*}$ treatment; ROI*time) or the between-subjects factors only (treatment*time) were carried out.

According to the aims of this study, three separate analyses were carried out. Paradigm 1 and paradigm 2 examined whether Homer1a expression may be significantly different among increasing doses of a second-generation multireceptor antipsychotic (i.e., asenapine) and a first-generation antipsychotic with almost selective D2R antagonist action (i.e., haloperidol). Accordingly, Homer1a expression was separately compared throughout asenapine-treated animals (asenapine groups, paradigm 1) and throughout haloperidol-treated animals (Haloperidol Groups, Paradigm 2). Paradigm 3 was aimed at evaluating whether multireceptor profile and substantial action on serotonin receptors may affect the cortical or striatal expression of Homer1a differentially. Accordingly, Homer1a expression was compared among the intermediate asenapine and haloperidol doses and a behaviorally compatible olanzapine dose, a multireceptor antipsychotic with a broader receptor profile but narrower action on serotonin receptors compared to asenapine.

Supplementary Materials: Supplementary materials can be found at http://www.mdpi.com/1422-0067/21/15/5555/s1.

Author Contributions: Methodology, F.I., E.F.B., A.d.B.; validation, A.d.B.; formal analysis, F.I., E.F.B.; investigation, F.I., E.F.B., C.A., A.B., L.V.; resources, A.E., A.d.B.; data curation, F.I.; writing一original draft preparation, F.I.; writing-review and editing, F.I., E.F.B., C.A., A.B., L.V., A.d.B., A.E.; supervision, A.d.B.; project administration, A.E., F.I.; funding acquisition, A.d.B. All authors have read and agreed to the published version of the manuscript.

Funding: This study was partially supported by a Lundbeck Italy $(0103379 / 2011)$ unrestricted grant to the Department of Neuroscience Reproductive and Odontostomatological Sciences, Laboratory of Molecular Psychiatry (Andrea de Bartolomeis). The funding source had no role in the study design; in the collection, analysis and interpretation of data; in the writing of the report; and in the decision to submit the article for publication.

Acknowledgments: The Authors wish to thank the whole staff of the Laboratory of Molecular and Translational Psychiatry and the Unit of Treatment Resistant Psychosis who aided and supported us in carrying out the procedures and the analyses reported in the present study.

Conflicts of Interest: Dr. Anna Eramo is an employee of Lundbeck LLC, Deerfield, IL, USA. All other Authors declare no potential conflict of interest.

\section{References}

1. Lieberman, J.A.; First, M.B. Psychotic Disorders. N. Engl. J. Med. 2018, 379, 270-280. [CrossRef]

2. Sahlholm, K.; Zeberg, H.; Nilsson, J.; Ogren, S.O.; Fuxe, K.; Arhem, P. The fast-off hypothesis revisited: A functional kinetic study of antipsychotic antagonism of the dopamine D2 receptor. Eur. Neuropsychopharmacol. 2016, 26, 467-476. [CrossRef]

3. De Bartolomeis, A.; Iasevoli, F.; Marmo, F.; Buonaguro, E.F.; Eramo, A.; Rossi, R.; Avvisati, L.; Latte, G.; Tomasetti, C. Progressive recruitment of cortical and striatal regions by inducible postsynaptic density transcripts after increasing doses of antipsychotics with different receptor profiles: Insights for psychosis treatment. Eur. Neuropsychopharmacol. 2015, 25, 566-582. [CrossRef]

4. Groos, D.; Zheng, F.; Rauh, M.; Quinger, B.; Kornhuber, J.; Muller, C.P.; Alzheimer, C. Chronic antipsychotic treatment targets GIRK current suppression, loss of long-term synaptic depression and behavioural sensitization in a mouse model of amphetamine psychosis. J. Psychopharmacol. 2018, 33, 74-85. [CrossRef] [PubMed]

5. De Bartolomeis, A.; Latte, G.; Tomasetti, C.; Iasevoli, F. Glutamatergic postsynaptic density protein dysfunctions in synaptic plasticity and dendritic spines morphology: Relevance to schizophrenia and other behavioral disorders pathophysiology, and implications for novel therapeutic approaches. Mol. Neurobiol. 2014, 49, 484-511. [CrossRef] [PubMed]

6. Iasevoli, F.; Tomasetti,C.; Buonaguro, E.F;; De Bartolomeis, A. The glutamatergic aspects of schizophrenia molecular pathophysiology: Role of the postsynaptic density, and implications for treatment. Curr. Neuropharmacol. 2014, 12, 219-238. [CrossRef] [PubMed]

7. Borovac, J.; Bosch, M.; Okamoto, K. Regulation of actin dynamics during structural plasticity of dendritic spines: Signaling messengers and actin-binding proteins. Mol. Cell. Neurosci. 2018, 91, 122-130. [CrossRef] [PubMed] 
8. Kaizuka, T.; Takumi, T. Postsynaptic density proteins and their involvement in neurodevelopmental disorders. J. Biochem. 2018, 163, 447-455. [CrossRef] [PubMed]

9. De Bartolomeis, A.; Buonaguro, E.F.; Latte, G.; Rossi, R.; Marmo, F.; Iasevoli, F.; Tomasetti, C. Immediate-Early Genes Modulation by Antipsychotics: Translational Implications for a Putative Gateway to Drug-Induced Long-Term Brain Changes. Front. Behav. Neurosci. 2017, 11, 240. [CrossRef]

10. Iasevoli, F.; Tomasetti, C.; Ambesi-Impiombato, A.; Muscettola, G.; De Bartolomeis, A. Dopamine receptor subtypes contribution to Homer1a induction: Insights into antipsychotic molecular action. Prog. Neuropsychopharmacol. Biol. Psychiatry 2009, 33, 813-821. [CrossRef]

11. Iasevoli, F.; Buonaguro, E.F.; Sarappa, C.; Marmo, F.; Latte, G.; Rossi, R.; Eramo, A.; Tomasetti, C.; De Bartolomeis, A. Regulation of postsynaptic plasticity genes' expression and topography by sustained dopamine perturbation and modulation by acute memantine: Relevance to schizophrenia. Prog. Neuropsychopharmacol. Biol. Psychiatry 2014, 54, 299-314. [CrossRef] [PubMed]

12. Buonaguro, E.F.; Iasevoli, F.; Marmo, F.; Eramo, A.; Latte, G.; Avagliano, C.; Tomasetti, C.; De Bartolomeis, A. Re-arrangements of gene transcripts at glutamatergic synapses after prolonged treatments with antipsychotics: A putative link with synaptic remodeling. Prog. Neuropsychopharmacol. Biol. Psychiatry 2017, 76, $29-41$. [CrossRef] [PubMed]

13. Meyer, D.; Bonhoeffer, T.; Scheuss, V. Balance and stability of synaptic structures during synaptic plasticity. Neuron 2014, 82, 430-443. [CrossRef] [PubMed]

14. Leber, S.L.; Llenos, I.C.; Miller, C.L.; Dulay, J.R.; Haybaeck, J.; Weis, S. Homer1a protein expression in schizophrenia, bipolar disorder, and major depression. J. Neural Transm. 2017, 124, 1261-1273. [CrossRef] [PubMed]

15. Spellmann, I.; Rujescu, D.; Musil, R.; Mayr, A.; Giegling, I.; Genius, J.; Zill, P.; Dehning, S.; Opgen-Rhein, M.; Cerovecki, A.; et al. Homer-1 polymorphisms are associated with psychopathology and response to treatment in schizophrenic patients. J. Psychiatr. Res. 2011, 45, 234-241. [CrossRef] [PubMed]

16. Szumlinski, K.K.; Lominac, K.D.; Kleschen, M.J.; Oleson, E.B.; Dehoff, M.H.; Schwarz, M.K.; Seeburg, P.H.; Worley, P.F.; Kalivas, P.W. Behavioral and neurochemical phenotyping of Homer1 mutant mice: Possible relevance to schizophrenia. Genes Brain Behav. 2005, 4, 273-288. [CrossRef]

17. Serchov, T.; Clement, H.W.; Schwarz, M.K.; Iasevoli, F.; Tosh, D.K.; Idzko, M.; Jacobson, K.A.; De Bartolomeis, A.; Normann, C.; Biber, K.; et al. Increased Signaling via Adenosine A1 Receptors, Sleep Deprivation, Imipramine, and Ketamine Inhibit Depressive-like Behavior via Induction of Homer1a. Neuron 2015, 87, 549-562. [CrossRef]

18. Serchov, T.; Schwarz, I.; Theiss, A.; Sun, L.; Holz, A.; Dobrossy, M.D.; Schwarz, M.K.; Normann, C.; Biber, K.; Van Calker, D. Enhanced adenosine A1 receptor and Homer1a expression in hippocampus modulates the resilience to stress-induced depression-like behavior. Neuropharmacology 2020, 162, 107834. [CrossRef]

19. Mereu, M.; Contarini, G.; Buonaguro, E.F.; Latte, G.; Manago, F.; Iasevoli, F.; De Bartolomeis, A.; Papaleo, F. Dopamine transporter (DAT) genetic hypofunction in mice produces alterations consistent with ADHD but not schizophrenia or bipolar disorder. Neuropharmacology 2017, 121, 179-194. [CrossRef]

20. Lum, J.S.; Pan, B.; Deng, C.; Huang, X.F.; Ooi, L.; Newell, K.A. Effects of short- and long-term aripiprazole treatment on Group I mGluRs in the nucleus accumbens: Comparison with haloperidol. Psychiatry Res. 2018, 260, 152-157. [CrossRef]

21. Clifton, N.E.; Cameron, D.; Trent, S.; Sykes, L.H.; Thomas, K.L.; Hall, J. Hippocampal Regulation of Postsynaptic Density Homer1 by Associative Learning. Neural Plast. 2017, 2017, 5959182. [CrossRef] [PubMed]

22. Datko, M.C.; Hu, J.H.; Williams, M.; Reyes, C.M.; Lominac, K.D.; Von Jonquieres, G.; Klugmann, M.; Worley, P.F.; Szumlinski, K.K. Behavioral and Neurochemical Phenotyping of Mice Incapable of Homerla Induction. Front. Behav. Neurosci. 2017, 11, 208. [CrossRef] [PubMed]

23. Buonaguro, E.F.; Morley-Fletcher, S.; Avagliano, C.; Vellucci, L.; Iasevoli, F.; Bouwalerh, H.; Van Camp, G.; Nicoletti, F.; Maccari, S.; De Bartolomeis, A. Glutamatergic postsynaptic density in early life stress programming: Topographic gene expression of mGlu5 receptors and Homer proteins. Prog. Neuropsychopharmacol. Biol. Psychiatry 2020, 96, 109725. [CrossRef] [PubMed]

24. Iasevoli, F.; Polese, D.; Ambesi-Impiombato, A.; Muscettola, G.; De Bartolomeis, A. Ketamine-related expression of glutamatergic postsynaptic density genes: Possible implications in psychosis. Neurosci. Lett. 2007, 416, 1-5. [CrossRef] [PubMed] 
25. De Bartolomeis, A.; Errico, F.; Aceto, G.; Tomasetti, C.; Usiello, A.; Iasevoli, F. D-aspartate dysregulation in Ddo(-/-) mice modulates phencyclidine-induced gene expression changes of postsynaptic density molecules in cortex and striatum. Prog. Neuropsychopharmacol. Biol. Psychiatry 2015, 62, 35-43. [CrossRef] [PubMed]

26. Clifton, N.E.; Trent, S.; Thomas, K.L.; Hall, J. Regulation and Function of Activity-Dependent Homer in Synaptic Plasticity. Mol. Neuropsychiatry 2019, 5, 147-161. [CrossRef]

27. De Bartolomeis, A.; Tomasetti, C. Calcium-dependent networks in dopamine-glutamate interaction: The role of postsynaptic scaffolding proteins. Mol. Neurobiol. 2012, 46, 275-296. [CrossRef]

28. Kammermeier, P.J. Surface clustering of metabotropic glutamate receptor 1 induced by long Homer proteins. BMC Neurosci. 2006, 7, 1. [CrossRef]

29. Shiraishi-Yamaguchi, Y.; Furuichi, T. The Homer family proteins. Genome Biol. 2007, 8, 206. [CrossRef]

30. Ronesi, J.A.; Huber, K.M. Homer interactions are necessary for metabotropic glutamate receptor-induced long-term depression and translational activation. J. Neurosci. 2008, 28, 543-547. [CrossRef]

31. Duncan, R.S.; Hwang, S.Y.; Koulen, P. Effects of Vesl/Homer proteins on intracellular signaling. Exp. Biol. Med. 2005, 230, 527-535. [CrossRef] [PubMed]

32. Bertaso, F.; Roussignol, G.; Worley, P.; Bockaert, J.; Fagni, L.; Ango, F. Homer1a-dependent crosstalk between NMDA and metabotropic glutamate receptors in mouse neurons. PLoS ONE 2010, 5, e9755. [CrossRef] [PubMed]

33. Iasevoli, F.; Ambesi-Impiombato, A.; Fiore, G.; Panariello, F.; Muscettola, G.; De Bartolomeis, A. Pattern of acute induction of Homerla gene is preserved after chronic treatment with first- and second-generation antipsychotics: Effect of short-term drug discontinuation and comparison with Homer1a-interacting genes. J. Psychopharmacol. 2011, 25, 875-887. [CrossRef] [PubMed]

34. Iasevoli, F.; Tomasetti, C.; Marmo, F.; Bravi, D.; Arnt, J.; De Bartolomeis, A. Divergent acute and chronic modulation of glutamatergic postsynaptic density genes expression by the antipsychotics haloperidol and sertindole. Psychopharmacology 2010, 212, 329-344. [CrossRef] [PubMed]

35. Tomasetti, C.; Dell'Aversano, C.; Iasevoli, F.; De Bartolomeis, A. Homer splice variants modulation within cortico-subcortical regions by dopamine D2 antagonists, a partial agonist, and an indirect agonist: Implication for glutamatergic postsynaptic density in antipsychotics action. Neuroscience 2007, 150, 144-158. [CrossRef] [PubMed]

36. Correll, C.U. From receptor pharmacology to improved outcomes: Individualising the selection, dosing, and switching of antipsychotics. Eur. Psychiatry 2010, 25 (Suppl. 2), S12-S21. [CrossRef]

37. Citrome, L. Asenapine review, part I: Chemistry, receptor affinity profile, pharmacokinetics and metabolism. Expert Opin. Drug Metab. Toxicol. 2014, 10, 893-903. [CrossRef]

38. Shahid, M.; Walker, G.B.; Zorn, S.H.; Wong, E.H. Asenapine: A novel psychopharmacologic agent with a unique human receptor signature. J. Psychopharmacol. 2009, 23, 65-73. [CrossRef]

39. Bymaster, F.P.; Calligaro, D.O.; Falcone, J.F.; Marsh, R.D.; Moore, N.A.; Tye, N.C.; Seeman, P.; Wong, D.T. Radioreceptor binding profile of the atypical antipsychotic olanzapine. Neuropsychopharmacology 1996, 14, 87-96. [CrossRef]

40. Kusumi, I.; Boku, S.; Takahashi, Y. Psychopharmacology of atypical antipsychotic drugs: From the receptor binding profile to neuroprotection and neurogenesis. Psychiatry Clin. Neurosci. 2015, 69, 243-258. [CrossRef]

41. Yano, M.; Beverley, J.A.; Steiner, H. Inhibition of methylphenidate-induced gene expression in the striatum by local blockade of D1 dopamine receptors: Interhemispheric effects. Neuroscience 2006, 140, 699-709. [CrossRef] [PubMed]

42. Kapur, S.; VanderSpek, S.C.; Brownlee, B.A.; Nobrega, J.N. Antipsychotic dosing in preclinical models is often unrepresentative of the clinical condition: A suggested solution based on in vivo occupancy. J. Pharmacol. Exp. Ther. 2003, 305, 625-631. [CrossRef] [PubMed]

43. McLean, S.L.; Neill, J.C.; Idris, N.F.; Marston, H.M.; Wong, E.H.; Shahid, M. Effects of asenapine, olanzapine, and risperidone on psychotomimetic-induced reversal-learning deficits in the rat. Behav. Brain Res. 2010, 214, 240-247. [CrossRef] [PubMed]

44. Ohyama, M.; Kondo, M.; Yamauchi, M.; Imanishi, T.; Koyama, T. Asenapine reduces anxiety-related behaviours in rat conditioned fear stress model. Acta Neuropsychiatr. 2016, 28, 327-336. [CrossRef]

45. Foute Nelong, T.; Manduca, J.D.; Zonneveld, P.M.; Perreault, M.L. Asenapine maleate normalizes low frequency oscillatory deficits in a neurodevelopmental model of schizophrenia. Neurosci. Lett. 2019, 711, 134404. [CrossRef] 
46. Crum, W.R.; Danckaers, F.; Huysmans, T.; Cotel, M.C.; Natesan, S.; Modo, M.M.; Sijbers, J.; Williams, S.C.; Kapur, S.; Vernon, A.C. Chronic exposure to haloperidol and olanzapine leads to common and divergent shape changes in the rat hippocampus in the absence of grey-matter volume loss. Psychol. Med. 2016, 46, 3081-3093. [CrossRef]

47. Franberg, O.; Marcus, M.M.; Ivanov, V.; Schilstrom, B.; Shahid, M.; Svensson, T.H. Asenapine elevates cortical dopamine, noradrenaline and serotonin release. Evidence for activation of cortical and subcortical dopamine systems by different mechanisms. Psychopharmacology 2009, 204, 251-264. [CrossRef]

48. Ambesi-Impiombato, A.; Panariello, F.; Dell'Aversano, C.; Tomasetti, C.; Muscettola, G.; De Bartolomeis, A. Differential expression of Homer 1 gene by acute and chronic administration of antipsychotics and dopamine transporter inhibitors in the rat forebrain. Synapse 2007, 61, 429-439. [CrossRef]

49. Iasevoli, F.; Fiore, G.; Cicale, M.; Muscettola, G.; De Bartolomeis, A. Haloperidol induces higher Homer1a expression than risperidone, olanzapine and sulpiride in striatal sub-regions. Psychiatry Res. 2010, 177, 255-260. [CrossRef]

50. Fatemi, S.H.; Reutiman, T.J.; Folsom, T.D.; Bell, C.; Nos, L.; Fried, P.; Pearce, D.A.; Singh, S.; Siderovski, D.P.; Willard, F.S.; et al. Chronic olanzapine treatment causes differential expression of genes in frontal cortex of rats as revealed by DNA microarray technique. Neuropsychopharmacology 2006, 31, 1888-1899. [CrossRef]

51. Sato, M.; Suzuki, K.; Nakanishi, S. NMDA receptor stimulation and brain-derived neurotrophic factor upregulate homer 1a mRNA via the mitogen-activated protein kinase cascade in cultured cerebellar granule cells. J. Neurosci. 2001, 21, 3797-3805. [CrossRef] [PubMed]

52. Zhang, G.C.; Mao, L.M.; Liu, X.Y.; Parelkar, N.K.; Arora, A.; Yang, L.; Hains, M.; Fibuch, E.E.; Wang, J.Q. In vivo regulation of Homer1a expression in the striatum by cocaine. Mol. Pharmacol. 2007, 71, 1148-1158. [CrossRef] [PubMed]

53. Nichols, C.D.; Garcia, E.E.; Sanders-Bush, E. Dynamic changes in prefrontal cortex gene expression following lysergic acid diethylamide administration. Brain Res. Mol. Brain Res. 2003, 111, 182-188. [CrossRef]

54. Moghaddam, B.; Bunney, B.S. Acute effects of typical and atypical antipsychotic drugs on the release of dopamine from prefrontal cortex, nucleus accumbens, and striatum of the rat: An in vivo microdialysis study. J. Neurochem. 1990, 54, 1755-1760. [CrossRef]

55. Kuroki, T.; Meltzer, H.Y.; Ichikawa, J. Effects of antipsychotic drugs on extracellular dopamine levels in rat medial prefrontal cortex and nucleus accumbens. J. Pharmacol. Exp. Ther. 1999, 288, 774-781.

56. Cahill, E.; Pascoli, V.; Trifilieff, P.; Savoldi, D.; Kappes, V.; Luscher, C.; Caboche, J.; Vanhoutte, P. $\mathrm{D} 1 \mathrm{R} / \mathrm{GluN1}$ complexes in the striatum integrate dopamine and glutamate signalling to control synaptic plasticity and cocaine-induced responses. Mol. Psychiatry 2014, 19, 1295-1304. [CrossRef]

57. Siddoway, B.; Hou, H.; Xia, H. Molecular mechanisms of homeostatic synaptic downscaling. Neuropharmacology 2014, 78, 38-44. [CrossRef]

58. Diering, G.H.; Nirujogi, R.S.; Roth, R.H.; Worley, P.F.; Pandey, A.; Huganir, R.L. Homer1a drives homeostatic scaling-down of excitatory synapses during sleep. Science 2017, 355, 511-515. [CrossRef]

59. Wang, Y.; Rao, W.; Zhang, C.; Zhang, C.; Liu, M.D.; Han, F.; Yao, L.B.; Han, H.; Luo, P.; Su, N.; et al. Scaffolding protein Homer1a protects against NMDA-induced neuronal injury. Cell Death Dis. 2015, 6, e1843. [CrossRef]

60. Naiker, D.V.; Catts, S.V.; Catts, V.S.; Bedi, K.S.; Bryan-Lluka, L.J. Dose determination of haloperidol, risperidone and olanzapine using an in vivo dopamine D2-receptor occupancy method in the rat. Eur. J. Pharmacol. 2006, 540, 87-90. [CrossRef]

61. Wadenberg, M.L.; Soliman, A.; VanderSpek, S.C.; Kapur, S. Dopamine D(2) receptor occupancy is a common mechanism underlying animal models of antipsychotics and their clinical effects. Neuropsychopharmacology 2001, 25, 633-641. [CrossRef]

62. Bymaster, F.; Perry, K.W.; Nelson, D.L.; Wong, D.T.; Rasmussen, K.; Moore, N.A.; Calligaro, D.O. Olanzapine: A basic science update. Br. J. Psychiatry Suppl. 1999, 174, 36-40. [CrossRef]

63. Turrone, P.; Remington, G.; Kapur, S.; Nobrega, J.N. Differential effects of within-day continuous vs. transient dopamine D2 receptor occupancy in the development of vacuous chewing movements (VCMs) in rats. Neuropsychopharmacology 2003, 28, 1433-1439. [CrossRef] [PubMed]

64. Valenti, O.; Cifelli, P.; Gill, K.M.; Grace, A.A. Antipsychotic drugs rapidly induce dopamine neuron depolarization block in a developmental rat model of schizophrenia. J. Neurosci. 2011, 31, 12330-12338. [CrossRef] [PubMed] 
65. Li, M. Antipsychotic-induced sensitization and tolerance: Behavioral characteristics, developmental impacts, and neurobiological mechanisms. J. Psychopharmacol. 2016, 30, 749-770. [CrossRef] [PubMed]

66. Seeman, P. All roads to schizophrenia lead to dopamine supersensitivity and elevated dopamine D2(high) receptors. CNS Neurosci. Ther. 2011, 17, 118-132. [CrossRef]

67. Gao, J.; Li, M. Time-dependence of risperidone and asenapine sensitization and associated D2 receptor mechanism. Behav. Brain Res. 2013, 257, 286-294. [CrossRef]

68. Gardoni, F.; Bellone, C. Modulation of the glutamatergic transmission by Dopamine: A focus on Parkinson, Huntington and Addiction diseases. Front. Cell. Neurosci. 2015, 9, 25. [CrossRef]

69. Li, M.; Fletcher, P.J.; Kapur, S. Time course of the antipsychotic effect and the underlying behavioral mechanisms. Neuropsychopharmacology 2007, 32, 263-272. [CrossRef]

70. Tappe, A.; Kuner, R. Regulation of motor performance and striatal function by synaptic scaffolding proteins of the Homer1 family. Proc. Natl. Acad. Sci. USA 2006, 103, 774-779. [CrossRef]

71. Errico, F.; Bonito-Oliva, A.; Bagetta, V.; Vitucci, D.; Romano, R.; Zianni, E.; Napolitano, F.; Marinucci, S.; Di Luca, M.; Calabresi, P.; et al. Higher free D-aspartate and N-methyl-D-aspartate levels prevent striatal depotentiation and anticipate L-DOPA-induced dyskinesia. Exp. Neurol. 2011, 232, 240-250. [CrossRef] [PubMed]

72. Zhang, Z.R.; Zhang, X.R.; Luan, X.Q.; Wang, X.S.; Wang, W.W.; Wang, X.Y.; Shao, B.; Xie, C.L. Striatal overexpression of beta-arrestin2 counteracts L-dopa-induced dyskinesia in 6-hydroxydopamine lesioned Parkinson's disease rats. Neurochem. Int. 2019, 131, 104543. [CrossRef] [PubMed]

73. Blandini, F.; Porter, R.H.; Greenamyre, J.T. Glutamate and Parkinson's disease. Mol. Neurobiol. 1996, 12, 73-94. [CrossRef] [PubMed]

74. Kapur, S. Psychosis as a state of aberrant salience: A framework linking biology, phenomenology, and pharmacology in schizophrenia. Am. J. Psychiatry 2003, 160, 13-23. [CrossRef] [PubMed]

75. Limongi, R.; Jeon, P.; Mackinley, M.; Das, T.; Dempster, K.; Theberge, J.; Bartha, R.; Wong, D.; Palaniyappan, L. Glutamate and Dysconnection in the Salience Network: Neurochemical, Effective Connectivity, and Computational Evidence in Schizophrenia. Biol. Psychiatry 2020, 88, 273-281. [CrossRef] [PubMed]

76. Liddle, E.B.; Price, D.; Palaniyappan, L.; Brookes, M.J.; Robson, S.E.; Hall, E.L.; Morris, P.G.; Liddle, P.F. Abnormal salience signaling in schizophrenia: The role of integrative beta oscillations. Hum. Brain Mapp. 2016, 37, 1361-1374. [CrossRef]

77. Yassa, M.A.; Stark, C.E. Multiple signals of recognition memory in the medial temporal lobe. Hippocampus 2008, 18, 945-954. [CrossRef]

78. Duan, X.; Hu, M.; Huang, X.; Su, C.; Zong, X.; Dong, X.; He, C.; Xiao, J.; Li, H.; Tang, J.; et al. Effect of Risperidone Monotherapy on Dynamic Functional Connectivity of Insular Subdivisions in Treatment-Naive, First-Episode Schizophrenia. Schizophr. Bull. 2020, 46, 650-660. [CrossRef]

79. Huang, M.; Li, Z.; Dai, J.; Shahid, M.; Wong, E.H.; Meltzer, H.Y. Asenapine increases dopamine, norepinephrine, and acetylcholine efflux in the rat medial prefrontal cortex and hippocampus. Neuropsychopharmacology 2008, 33, 2934-2945. [CrossRef]

80. Marston, H.M.; Young, J.W.; Martin, F.D.; Serpa, K.A.; Moore, C.L.; Wong, E.H.; Gold, L.; Meltzer, L.T.; Azar, M.R.; Geyer, M.A.; et al. Asenapine effects in animal models of psychosis and cognitive function. Psychopharmacology 2009, 206, 699-714. [CrossRef]

81. Tarazi, F.I.; Moran-Gates, T.; Wong, E.H.; Henry, B.; Shahid, M. Differential regional and dose-related effects of asenapine on dopamine receptor subtypes. Psychopharmacology 2008, 198, 103-111. [CrossRef] [PubMed]

82. Choi, Y.K.; Wong, E.H.; Henry, B.; Shahid, M.; Tarazi, F.I. Repeated effects of asenapine on adrenergic and cholinergic muscarinic receptors. Int. J. Neuropsychopharmacol. 2010, 13, 405-410. [CrossRef] [PubMed]

83. Banasikowski, T.J.; Beninger, R.J. Haloperidol conditioned catalepsy in rats: A possible role for D1-like receptors. Int. J. Neuropsychopharmacol. 2012, 15, 1525-1534. [CrossRef]

84. Ene, H.M.; Kara, N.Z.; Barak, N.; Ben-Mordechai, T.R.; Einat, H. Effects of repeated asenapine in a battery of tests for anxiety-like behaviours in mice. Acta Neuropsychiatr. 2016, 28, 85-91. [CrossRef] [PubMed]

85. Paxinos, G.; Watson, C. The Rat Brain in Stereotaxic Coordinates, 6th ed.; Academic Press Inc.: Sidney, Australia, 2007.

(C) 2020 by the authors. Licensee MDPI, Basel, Switzerland. This article is an open access article distributed under the terms and conditions of the Creative Commons Attribution (CC BY) license (http://creativecommons.org/licenses/by/4.0/). 